\title{
Pro- and Antioxidant Functions of the Peroxisome-Mitochondria Connection and Its Impact on Aging and Disease
}

\author{
Amparo Pascual-Ahuir, ${ }^{1}$ Sara Manzanares-Estreder, ${ }^{1,2}$ and Markus Proft ${ }^{2}$ \\ ${ }^{1}$ Department of Biotechnology, Instituto de Biología Molecular y Celular de Plantas, Universitat Politècnica de València-CSIC, \\ Ingeniero Fausto Elio s/n, 46022 Valencia, Spain \\ ${ }^{2}$ Department of Molecular and Cellular Pathology and Therapy, Instituto de Biomedicina de Valencia IBV-CSIC, Jaime Roig 11, \\ 46010 Valencia, Spain
}

Correspondence should be addressed to Markus Proft; mproft@ibv.csic.es

Received 28 April 2017; Accepted 27 June 2017; Published 24 July 2017

Academic Editor: Marina Sokovic

Copyright (c) 2017 Amparo Pascual-Ahuir et al. This is an open access article distributed under the Creative Commons Attribution License, which permits unrestricted use, distribution, and reproduction in any medium, provided the original work is properly cited.

\begin{abstract}
Peroxisomes and mitochondria are the main intracellular sources for reactive oxygen species. At the same time, both organelles are critical for the maintenance of a healthy redox balance in the cell. Consequently, failure in the function of both organelles is causally linked to oxidative stress and accelerated aging. However, it has become clear that peroxisomes and mitochondria are much more intimately connected both physiologically and structurally. Both organelles share common fission components to dynamically respond to environmental cues, and the autophagic turnover of both peroxisomes and mitochondria is decisive for cellular homeostasis. Moreover, peroxisomes can physically associate with mitochondria via specific protein complexes. Therefore, the structural and functional connection of both organelles is a critical and dynamic feature in the regulation of oxidative metabolism, whose dynamic nature will be revealed in the future. In this review, we will focus on fundamental aspects of the peroxisome-mitochondria interplay derived from simple models such as yeast and move onto discussing the impact of an impaired peroxisomal and mitochondrial homeostasis on ROS production, aging, and disease in humans.
\end{abstract}

\section{Introduction}

Oxidative stress is causally linked to accelerated aging and aging-related diseases [1]. In eukaryotic cells, mitochondria and peroxisomes are the main ROS contributors $[2,3]$. At the same time, both organelles are equipped with their own ROS scavenging repertoire of enzymes. Mitochondria and peroxisomes are metabolically linked because they share common pathways such as the fatty acid $\beta$-oxidation. However, both organelles are much more intimately regulated with respect to ROS homeostasis and the clearance of dysfunctional organelles. Here, we will focus on our current knowledge of mitochondrial and peroxisomal pro- and antioxidant functions, their coordinated turnover by autophagic pathways, and their functional and physical interactions. Investigation in the yeast model is contributing fundamental insights into the mechanisms of quality control of peroxisomes and mitochondria. Therefore, we will compare the dynamics of both organelles and its impact on cell survival and health in yeast and mammalian cells throughout the review.

\section{Pro- and Antioxidant Functions of Peroxisomes}

Peroxisomes are versatile organelles found in most eukaryotic cells. The name peroxisome has been originally introduced for a cellular organelle which contains at least one $\mathrm{H}_{2} \mathrm{O}_{2}$-producing oxidase and the $\mathrm{H}_{2} \mathrm{O}_{2}$-detoxifying enzyme catalase [4]. Today, we know that peroxisomal function goes far beyond the metabolism of reactive oxygen species. Peroxisomes contain more than 50 different enzymatic activities, which shape important metabolic and anabolic functions, including fatty acid oxidation and lipid biosynthesis. As they share an oxidative metabolism together with mitochondria, they are key organelles in the cellular homeostasis of ROS 


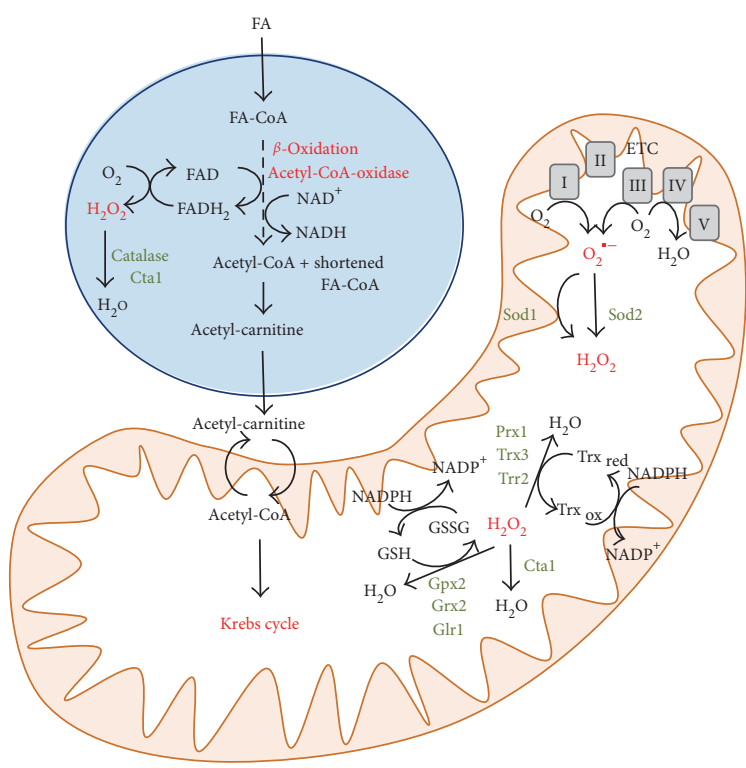

(a) Yeast

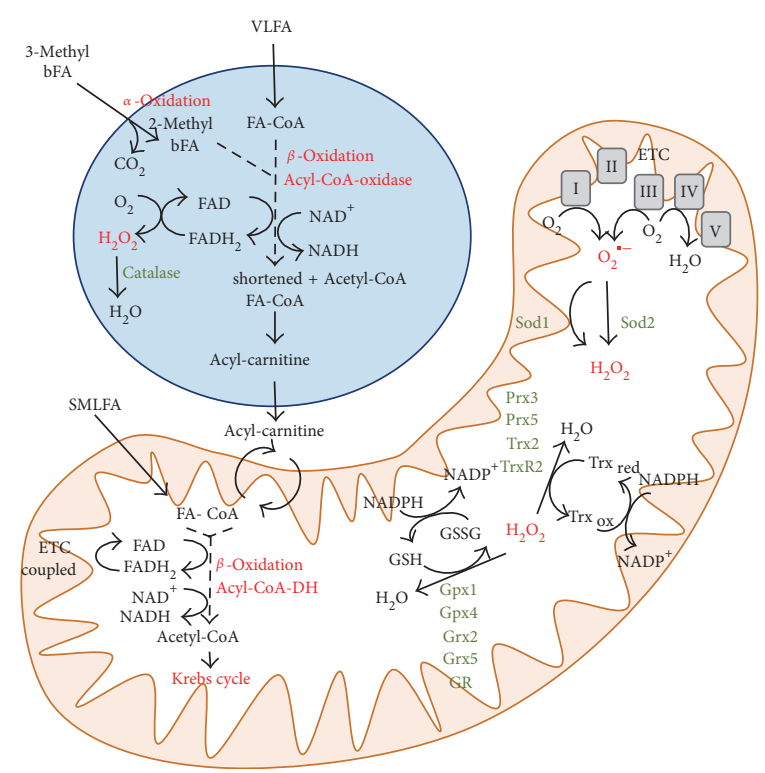

(b) Mammalian cells

Figure 1: Pro- and antioxidant functions of peroxisomes and mitochondria in yeast and mammalian cells. Peroxisomes play important roles in the oxidative degradation of fatty acids. The peroxisomal fatty acid oxidation pathways are schematically depicted for budding yeast (a) and mammalian cells (b). Mitochondrial pro- and antioxidant functions and their interconnection with peroxisomes are summarized. ROS-generating and ROS-scavenging functions are highlighted for both organelles. FA = fatty acid; bFA=branched fatty acid; VLFA = very long fatty acid; SMLFA = small, medium, and long fatty acids; FA-CoA = fatty acyl coenzyme A; ETC=electron transport chain; Gpx=glutathione peroxidase; Grx=glutaredoxin; GR and Glr=glutathione reductase; Sod=superoxide dismutase; $\mathrm{GSH}=$ reduced glutathione; GSSG = oxidized glutathione; $\mathrm{Prx}=$ peroxiredoxin; $\operatorname{Trx}=$ thioredoxin; $\operatorname{Trr}$ and $\operatorname{TrxR}=$ thioredoxin reductase; $\mathrm{DH}=$ dehydrogenase; I to $\mathrm{V}=$ mitochondrial respiratory complexes I-V.

$[5,6]$. Although the variability of the metabolic activities contained by peroxisomes of different organisms or tissues is considerable, their universal functions are the oxidative metabolism of fatty acids and the degradation of $\mathrm{H}_{2} \mathrm{O}_{2}$ by catalases [7]. Thus, peroxisomes are both sources and sinks of ROS and account for a great part of oxygen consumption and ROS production (up to 35\%) in metabolically active tissues such as the liver $[4,8]$. Peroxisomes are much more dynamic organelles than anticipated, and the impact of the dynamic regulation of their size, abundance, morphology, and function in response to environmental stimuli or during development has only recently been addressed [9-11]. Given the dynamic nature of peroxisomes and their central role in fatty acid oxidation, an efficient quality control of this organelle is required to avoid oxidative damage and premature aging $[3,5,12]$.

The oxidative degradation of fatty acids takes place in peroxisomes and mitochondria (Figure 1). However, the compartmentalization of fatty acid oxidation pathways between these two organelles is variable in different organisms. Budding yeast, for example, degrades fatty acids exclusively in peroxisomes via $\beta$-oxidation, which implies the removal of two carbons from the fatty acid carboxyl terminus [13]. The first step in this process is catalyzed by acyl-CoA oxidases, which reduce $\mathrm{O}_{2}$ to $\mathrm{H}_{2} \mathrm{O}_{2}$. The end product of $\beta$ oxidation in yeast, acetyl-CoA, is transformed to acetylcarnitine by peroxisomal carnitine acetyl-CoA transferases and shuttled into mitochondria to fuel the Krebs cycle and respiration. In human cells, mitochondria and peroxisomes cooperate in different routes of fatty acid oxidation [14]. Here, fatty acids with shorter chain lengths $\left(\mathrm{C}_{6}-\mathrm{C}_{20}\right)$ are oxidized by $\beta$-oxidation in mitochondria, where the first step is catalyzed by acyl-CoA dehydrogenases coupled to the mitochondrial electron transport and not to $\mathrm{H}_{2} \mathrm{O}_{2}$ production. However, fatty acids with longer chain lengths $\left(>\mathrm{C}_{20}\right)$ or dicarboxylic fatty acids have to be degraded by the peroxisomal $\beta$-oxidation pathway. Additionally, 3-methyl-branched fatty acids are exclusively metabolized in human peroxisomes by the process of $\alpha$-oxidation, which represents an additional source of the ROS $\mathrm{H}_{2} \mathrm{O}_{2}$ originating from these organelles. In the human case, $\beta$-oxidation is not necessarily carried out to completion in peroxisomes; hence, the final product is a shortened fatty acyl-CoA, which is shuttled to mitochondria in the form of the corresponding fatty acyl carnitine conjugate. It is worth noting that ROS production by mammalian peroxisomes is not limited to the fatty acyl-CoA oxidases of the $\beta$-oxidation pathway. Additionally, oxidases participating in glyoxylate metabolism, amino acid catabolism, or the oxidation of polyamines are potential ROS sources of the peroxisome [15].

Peroxisomes contain several antioxidant systems, which are important for ROS homeostasis of this highly oxidative organelle (Figure 1). Since the $\beta$-oxidation pathway directly produces $\mathrm{H}_{2} \mathrm{O}_{2}$, the detoxifying catalase activity is of central importance for the redox balance of the organelle $[16,17]$. It has been shown in yeast that peroxisomal catalase is 
required also for the tolerance to externally added $\mathrm{H}_{2} \mathrm{O}_{2}$, which suggests that peroxisomes are generally important for cellular ROS detoxification [18]. An additional peroxisomal antioxidant activity has been described in yeast as the glutathione peroxidase Gpxl, whose activity is generally required for a proper peroxisomal biogenesis [19]. Also in mammalian cells, catalase is the predominant antioxidant activity of peroxisomes. However, several other ROS detoxifying are known in higher eukaryotes to contribute to peroxisomal redox balance, which include superoxide dismutases, peroxiredoxins, glutathione S-transferases, and epoxide hydrolases [5].

The importance of peroxisomes in the maintenance of a healthy redox balance has been derived from situations, which interfere with a normal peroxisomal function and biogenesis. Artificial stimulation of peroxisomal biogenesis by the long-term exposure to peroxisomal proliferators causes oxidative liver damage and ROS imbalances in general in rodents $[20,21]$, most likely by the uncompensated induction of ROS-generating peroxisomal enzymes [22]. However, stimulated peroxisomal proliferation seems to reduce ROS levels in murine neurons [23]. Moreover, the loss of peroxisomes increases the apoptotic damage in the cerebellum and neurodegeneration [24, 25], and a deficiency in human catalase activity increases the susceptibility to aging-related diseases such as diabetes, cancer, or atherosclerosis [26]. Moreover, catalase mutants show phenotypes of accelerated aging in the Caenorhabditis model [27]. In yeast, loss of catalase function modulates the lifespan depending on the metabolic activity of peroxisomes [18]. Thus, peroxisomal (together with mitochondrial) activity in maintaining the cellular redox state is generally important for cell survival and health $[28,29]$.

\section{Pro- and Antioxidant Functions of Mitochondria}

Mitochondria have been traditionally described as the cellular power-house as they are the main contributors of energy through oxidative phosphorylation and the biosynthesis of ATP. Structurally, they contain two biologically different membrane systems: the outer membrane containing the intermembrane space and the inner membrane containing the mitochondrial matrix. The different electron transport chain (ETC) protein complexes are embedded in the inner membrane, which is heavily folded into the cristae structure [30]. Apart from regulating the energy metabolism of the eukaryotic cell, mitochondria are involved in many other fundamental processes, such as the control of the cell cycle or the induction or prevention of cell death [31-33].

Generation of ATP by the mitochondrial oxidative phosphorylation is based on the reduction of molecular oxygen, which can produce toxic byproducts such as the superoxide radical $\left(\mathrm{O}_{2}^{-}\right)$or hydrogen peroxide $\left(\mathrm{H}_{2} \mathrm{O}_{2}\right)$. Only complex IV (cytochrome $c$ oxidase) of the ETC is able to fully reduce molecular oxygen to water and therefore has an antioxidant function in the ETC, while mainly complex I (NADH ubiquinone oxidoreductase) and complex III (ubiquinol-cytochrome $c$ oxidoreductase) contribute to superoxide radical formation due to incomplete $\mathrm{O}_{2}$ reduction [34]. These ROS can trigger the formation of even more harmful secondary ROS such as the hydroxyl radical $(\cdot \mathrm{OH})$, which are supposed to be responsible for the main oxidative damage derived from mitochondrial activity $[35,36]$. Electron leakage from mitochondrial electron transport chains is now recognized as one of the most important intracellular sources of ROS including hydroxyl radicals [37]. Mitochondria possess their own antioxidant systems, which consist of various ROS-scavenging enzymes and low molecular antioxidants $[38,39]$. Primary antioxidant enzymes are superoxide dismutases (SOD), which reduce superoxide radicals to hydrogen peroxide. Two types of SOD can be found in mammalian mitochondria: manganese superoxide dismutase (SOD2) in the mitochondrial matrix and copper/zinc superoxide dismutase (SOD1) in the intermembrane space [40-42]. Detoxification of mitochondrial $\mathrm{H}_{2} \mathrm{O}_{2}$ can be achieved by the activity of glutathione peroxidases (GPx) and glutaredoxins (GRx). At least two GPx isoforms have been identified in the mammalian mitochondria, GPx1 and GPx4 [43, 44], and two Grx isoenzymes are active in mitochondria, Grx2 and Grx5 [45]. During this reaction, glutathione is oxidized, which has to be reduced by mitochondrial glutathione reductase (GR) with the help of the mitochondrial NADPH pool. Additionally, hydrogen peroxide can be reduced by mitochondrial peroxiredoxins (Prx) coupled to the oxidation of thioredoxins (Trx), which are finally recycled at the expense of NADPH by Trx reductases (TrxR). In the mammalian mitochondria, at least the isoenzymes Prx3, Prx5, and Trx2 have been described [46-50]. Among the nonenzymatic antioxidants found in the mammalian mitochondria, the high $\mathrm{NADPH}$ pool, vitamin $\mathrm{C}$, and coenzyme $\mathrm{Q}$ have been reported with important functions in ROS elimination [51-53]. As opposed to peroxisomes, significant catalase activity is absent from the mammalian mitochondria. Yeast mitochondria also contain a full set of antioxidant systems based on glutathione or thioredoxin with the enzymes Gpx2, Grx2, Glr1, Prx1, Trx3, and Trr2 [54]. Also in yeast, the Sod $1 \mathrm{Cu} / \mathrm{Zn}$ - and the Sod2 manganese-superoxide dismutases are the primary ROS-reducing enzymes in mitochondria $[55,56]$. Additionally, and different to the situation in mammalian cells, the peroxisomal catalase Ctal can also be found inside the yeast mitochondria [57].

The free radical theory of aging first postulated that the accumulation of cellular damage caused by free radicals was a decisive driving force in the process of aging and in the determination of a lifespan [58]. A major prediction derived from this theory was that the supplementation with antioxidants or genetic manipulation of endogenous ROSscavenging systems would have a beneficial effect on the lifespan of the organism. However, no conclusive results were obtained by studies of this type in general [59-64], which led to a revision of the original hypothesis in a form of the mitochondrial-free radical theory [65]. Here, mitochondrially generated free radicals were supposed to have a major effect on aging, and thus it was proposed that reinforcement of mitochondrial antioxidant systems had lifespan extension effects. On the contrary, mitochondrial damage or insufficient ROS-scavenging activity of mitochondria could lead 
to lifespan shortening. A large body of evidence has accumulated, which supports this hypothesis [66-68]. Artificial targeting of catalase to mitochondria extends the lifespan of mice [69]. Similarly, the mitochondria-targeted antioxidant SkQ1 has lifespan extension effects in several rodent models [70]. On the contrary, mouse models, with mutations in the mitochondrial DNA polymerase gamma, have a shorter lifespan and manifest several phenotypes of accelerated aging [71]. Furthermore, the accumulation of damage in the mitochondrial DNA (mtDNA) caused in these mouse models has been reported to favor apoptosis and age-related diseases [72] and can be reverted by mitochondria-targeted catalase [73]. Moreover, deficiencies in Mn-superoxide dismutase activity increases oxidative stress and promotes aging in Drosophila [74-76]. Yeast has very efficiently contributed to reveal physiologically relevant determinants of lifespan and health span in higher organisms $[77,78]$. Also in this simple model, mitochondria have been identified with an important role in the establishment of lifespan [79]. Specifically, yeast sod mutants are known to critically interfere with longevity [80]. Having found that mitochondrial ROS production is a driving factor of aging, a growing number of studies now intend to reduce ROS production by the targeted delivery of antioxidants to the mitochondria [1]. These approaches include the use of mitochondria-directed redox agents to detoxify mitochondrial ROS [81] or modulators of mitochondrial electron transport and leakage to avoid mitochondrial ROS [82]. These strategies are beyond the scope of this review; hence, the interested reader might consult summaries made by excellent recent revisions [68, 83, 84].

It is important to note that ROS might not always trigger fatal oxidative damage within a cell. On the contrary, low ROS concentrations have important biological functions related to signaling and stress resistance [85-87]. It has been increasingly clear in the last years that oxidative stress is able to actually promote longevity, which has led to the theory of mitochondrial hormesis. Here, stimuli such as calorie restriction or exercise are supposed to trigger adaptations, which lead to the reinforcement of endogenous antioxidant systems, increased stress tolerance, and subsequently slow down the aging process $[88,89]$. There are many experimental findings in different model systems including yeast, Caenorhabditis or Drosophila, which are in line with the mitohormesis hypothesis $[90,91]$. Inhibition of respiration, for example, increases the lifespan of nematodes dependent on the production of mitochondrial ROS [92]. Calorie restriction and especially glucose restriction partially induce mitochondrial metabolism and prevent aging from yeast to flies $[93,94]$. It has been demonstrated in Caenorhabditis that reduced sugar intake induces mitochondrial respiration and ROS production, which are necessary for lifespan extension, and this beneficial effect is reverted by antioxidants [95]. In yeast, several interventions including the inhibition of nutrient-sensing protein kinases or dietary restriction cause an extended lifespan [96]. These effects have been shown to depend on mitochondrial activity in general or specific mitochondrial ROS-scavenging activities [97-100]. Taken together, mitochondrial ROS balance is decisive for the modulation of longevity and we will continue to summarize how mitochondrial and peroxisomal activity might change during the process of aging and in age-related diseases.

\section{Compromised Function of Peroxisomes and Mitochondria during Aging}

The investigation of the detrimental function of peroxisomal dysfunction in the process of aging has traditionally lagged behind mitochondria, which were considered the major source of oxidative stress-related senescence. However, it is much more considered now that a misregulated peroxisomal ROS balance is important in the occurrence of age-related diseases $[3,101,102]$. Recently, it has been shown that a deficiency in peroxisomal ROS clearance actually affects mitochondrial function. Specifically, inhibition of peroxisomal catalase activity negatively modulates the redox balance in mouse mitochondria [103] and human fibroblast [104]. Moreover, a localized oxidative damage to peroxisomes leads to mitochondrial fragmentation and cell death $[105,106]$. These results reflect the intimate relation of both organelles in the maintenance of the cellular redox balance and furthermore suggest that peroxisomal dysfunction can subsequently damage mitochondria. Therefore, peroxisomes can act at the forefront in the initiation of cellular oxidative damage and aging [3]. Accordingly, several reports support that peroxisomal function continuously declines during aging. In cultured human cells, it has been shown that catalase is increasingly excluded from peroxisomes after repeated cell passage. At the same time, old cells accumulate more dysfunctional peroxisomes which raise cellular ROS levels and ultimately might accelerate aging [107]. In line with these results is the finding that in Caenorhabditis the abundance of many peroxisomal proteins decays during aging. Importantly, this affects the Pex 5 importer of peroxisomal matrix proteins [108]. Pex5 function has been found to be regulated by the redox state of the organelle $[109,110]$. Thus, the decline of peroxisomal protein import by oxidative stress, including the import of antioxidants, might be a trigger for senescence. Additionally, it has been shown that yeast peroxisome proliferation is regulated by the redox state of the organelle [19]. Oxidative stress might inhibit yeast peroxisomal biogenesis via multiple targets, such as Pex 5 or Pex11 [109, 111-113]. Finally, interference with peroxisomal fission increases the lifespan of yeast cells [114]. Taken together, maintaining functional peroxisomes emerges as an important determinant of the cellular lifespan. The relevant mechanisms of peroxisomal homeostasis will be discussed in the following section.

A large body of experiments has demonstrated that mitochondrial dysfunction is an important trigger for cellular senescence, which contributes to aging in addition to other prosenescence stimuli [115-118]. Although it is clear that dysfunctional mitochondria accumulate in senescent cells and that this is a major driving force for accelerated aging [119-121], it is less understood how mitochondria become dysfunctional during the aging process $[122,123]$. Mitochondrial defects which accumulate in older cells range from an increased mitochondrial mass, a decrease of respiratory coupling, less efficient ATP production during respiration, loss 
of respiratory complex I function, and increased mitochondrial ROS production $[117,118]$.

Importantly, there is increasing evidence that misregulated ROS production is an important trigger for mitochondrial dysfunction in the context of several age-related diseases. Cardiomyopathy in the old age, for example, is accompanied by an impairment of oxidative phosphorylation in heart mitochondria $[87,124]$. Consequently, the rate of ROS production increases with age especially in cardiac mitochondria [125]. Similarly, an inefficient mitochondrial energy metabolism has been linked to cardiovascular diseases $[126,127]$. In order to explain this decline in mitochondrial function, several mechanisms have been suggested, for example, an impaired biogenesis of the organelle, increased mitochondrial uncoupling, or the accumulation of mt-DNA mutations [128-130]. Mitochondrial dysfunction has been broadly implied in age-related neurodegenerative diseases [131-133]. In the particular case of Alzheimer's disease, the accumulation of the toxic aggregates of the unprocessed $\beta$ amyloid peptide is known to inhibit the mitochondrial electron transport chain, cause ROS overproduction, and induce mitochondria-mediated cell death [134-138]. Additionally, the accumulation of mt-DNA aberrations and failures in the dynamic regulation of mitochondrial morphology by fission have been linked to Alzheimer's disease [139-141]. Parkinson's disease is another neurodegenerative disorder where mitochondrial dysfunction has been identified as a major driving force. Several genes have been associated with familial Parkinson, and most of these PARK loci are functionally related with mitochondria [142]. PARK1 for example encodes $\alpha$-synuclein, which can inhibit mitochondrial fusion [143]. Mutations in PARK7 affect DJ-1 function causing defects of mitochondrial respiratory complex I, ROS overproduction, and a loss of mitochondrial membrane potential $[144,145]$. PARK8 encodes the LRRK2 protein kinase, whose function is needed for proper oxidative phosphorylation activity and mitochondrial fission [146, 147]. PARK2 and PARK6 encode Parkin and PINK1 (PTENinduced kinase 1), which are involved in the correct turnover and degradation of dysfunctional mitochondria by mitophagy $[148,149]$. The mechanisms of autophagic removal of excess or malfunctioning mitochondria and peroxisomes will be described in the following section.

Mitochondria form dynamic networks, whose morphology changes in response to stress and nutritional stimuli and damage [150, 151]. Several independent experimental evidences exist showing that changes in mitochondrial dynamics are intimately linked to senescence and accelerated aging [152]. During aging, mitochondrial fission is reduced, which leads to mitochondrial elongation [153]. It has been shown that inhibition of the profission protein FIS1 or the E3 ubiquitin ligase MARCH5, which positively regulates mitochondrial fission, induces senescence via mitochondrial elongation $[154,155]$. In yeast, the pharmacological repression of mitochondrial fission leads to a lifespan extension $[154,156]$. These results indicate that mitochondrial fission is needed for a normal cellular lifespan. This is in agreement with the finding that hyperelongated mitochondria have lower membrane potentials and excessively produce ROS
$[153,154]$. Additionally, the reinforcement of mitochondrial fission by the overexpression of FIS1 can reduce age-related phenotypes [153]. Importantly, mitochondrial fission is needed for efficient clearance of defective mitochondria via mitophagy, which could additionally explain the antiaging effect of the fission process $[157,158]$. In a reverse manner, oxidative stress alters mitochondrial morphology via the modulation of several proteins involved in mitochondrial dynamics and this phenomenon has been linked to cancer progression [159-161]. Exogenous ROS efficiently inhibit mitofusins (Mfn1 and Mfn2) in human fibroblasts and muscle myoblasts inducing mitochondrial fission and membrane depolarization $[162,163]$. In yeast, oxidative stress promotes mitochondrial fragmentation via the stimulation of the assembly of the mitochondrial fission machinery composed of Mdv1, the Dnm1 GTPase, and the Fis1 mitochondrial fission proteins [164].

Another important question is whether the asymmetric inheritance of mitochondria of different qualities or activities modulates the aging process. This phenomenon has been extensively studied in the yeast model of replicative aging [165]. During cell divisions, proaging components such as damaged or dysfunctional mitochondria are retained in the older mother cell and not transmitted to the young daughter cell [166]. Specific mitochondrial tether proteins such as Mmrl have been identified, which actively retain highly oxidative mitochondria in the mother cell. Consequently, interference with this asymmetric mitochondrial distribution by deletion of Mmr1 shortens the yeast lifespan [167]. Additional anchor proteins such as Mfb1 have been identified now, which guarantee the remaining of highly functional mitochondria in the mother cell [168]. Interestingly, the unequal distribution of mitochondria from the mother to daughter cell is conditioned by mitochondrial dynamics since it is impaired in mitochondrial fusion mutants [169]. Taken together, the fusion/fission process of mitochondria (and peroxisomes) is important for the maintenance of a healthy redox balance and lifespan. The quality control of both organelles via autophagic mechanisms is of key importance to maintain both mitochondria and peroxisomes functional.

\section{Quality Control of Peroxisomes and Mitochondria by Autophagy and the Impact on Health}

The number, activity, and quality of peroxisomes are dynamically adapted and controlled in the cell [170]. An important mechanism to eliminate dysfunctional or superfluous peroxisomes consists in a specific autophagy termed pexophagy [171]. The mechanisms which assure the engulfment of specific peroxisomes in autophagosomes and their subsequent degradation in vacuoles (fungi) or lysosomes (mammals) have been especially elucidated in the yeast model [172]. Here, pexophagy has been intensively studied in response to nutritional changes from respiratory to fermentative growth conditions, which implies the proteolytic removal of excess peroxisomes [172]. The key step in the initiation of 

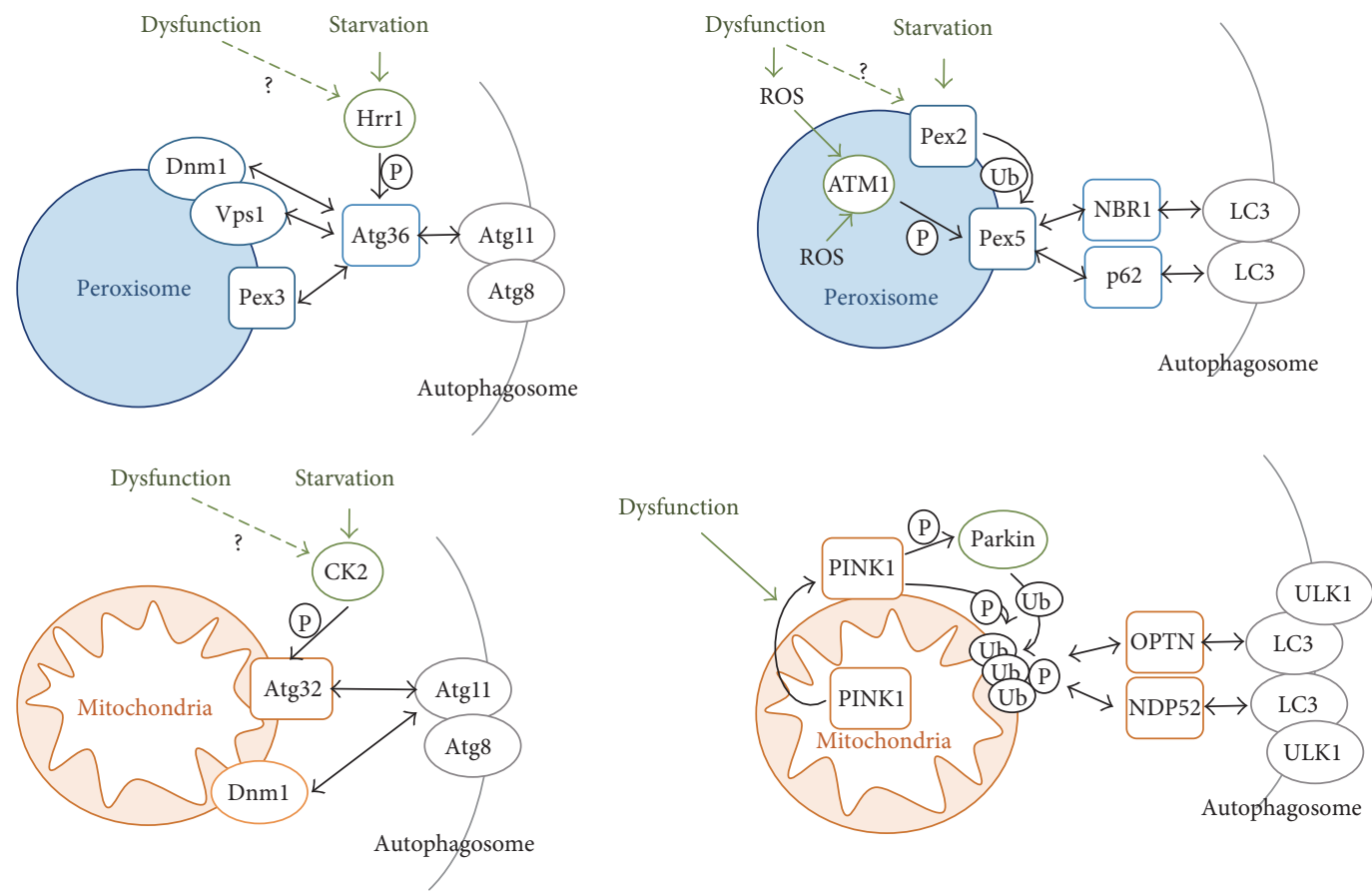

(a) Yeast

(b) Mammalian cells

FIGURE 2: Mechanisms of autophagic removal of peroxisomes and mitochondria. In the upper panel, pexophagy mechanisms are depicted for budding yeast (a) and mammalian cells (b). In yeast, pexophagy is induced by the Hrr1 kinase which phosphorylates the Atg36 adaptor. Atg36 contacts the Pex3 peroxisomal receptor, the fission machinery (Dnm1, Vps1), and the autophagosomal adaptor Atg11. In mammalian cells, a dysfunctional peroxisome and general ROS increase activate the ATM kinase, which phoshorylates the Pex5 receptor. Pex5 is additionally targeted by ubiquitination via the starvation-inducible Pex2. Modified Pex5 interacts with the autophagosomal adaptors NBR1 and p62. In the lower panel, mitophagy mechanisms are depicted for budding yeast (left) and mammalian cells (right). In yeast, starvation induces mitophagy via casein kinase 2 (CK2), which phosphorylates the Atg32 receptor. Modified Atg32 interacts with the Atg11 autophagosomal adaptor, which also contacts the mitochondrial fission machinery (Dnm1). In mammalian cells, mitochondrial dysfunction triggers the exposure of PINK1 at the organelle surface. PINK1 phosphorylates and activates the Parkin ubiquitin ligase, which marks outer mitochondrial membrane proteins. PINK1 additionally phosphorylates polyubiquitin chains at mitochondria, which leads to recognition by the autophagosomal adaptor proteins Optineurin (OPTN) and NDP52. P= phosphorylation; Ub= ubiquitination.

pexophagy is the formation of a preautophagosomal structure at the surface of the organelle [173-176] (Figure 2). Several Atg proteins are coordinated in this process termed cytoplasm-to-vacuole targeting; however, the Atg11 cytosolic adaptor protein is the physical link of the organelle to be degraded and the autophagosome in the case of both peroxisomes and mitochondria $[175,176]$. Atg11 contacts the peroxisome via a specific adaptor protein at the peroxisomal surface. In budding yeast, the specific adaptor for pexophagy is Atg36, which directly interacts with the peroxisomal Pex3 protein and the core phagosomal components Atg11 and Atg8 [177, 178]. One way to induce pexophagy is through the phosphorylation of Atg36 by the Hrr25 casein kinase homolog [179]. Hrr25 phosphorylates a specific serine residue (S97) in the Atg36 adaptor, which triggers its interaction with Atg11 but not with Pex3 [179]. Therefore, phosphorylation of Atg36 by Hrr25 is a switch in the initiation of pexophagy by allowing the interaction of the peroxisome with the central autophagosomal machinery [179]. Peroxisomes have their own fission system to allow an autonomous proliferation of the organelle. In yeast, the dynamin-related small GTPases Dnm1 and Vps1 together with the fission protein
Fis1 are required for peroxisomal fission [180-182]. It has been shown recently that peroxisomal fission is required for pexophagy [183]. Moreover, the Atg11 adaptor protein interacts directly with Dnm1 and Vps1 [183]. Taken together, the Atg11 mediated recruitment of the preautophagosomal structure at strategic sites of the peroxisome via Pex3, and the fission machinery seems to be the initial step for marking peroxisomes for proteolytic degradation. It is less understood whether and how pexophagy serves to eliminate dysfunctional peroxisomes. However, it has been shown in yeast that artificially created catalase aggregates are cleared in a process that depends on peroxisomal fission and pexophagy [184]. Additionally, peroxisomal protein import defects have been shown to increase the rate of pexophagy [185]. These results suggest that the autophagic removal of damaged peroxisomes with increased ROS production could be an important mechanism to maintain a correct redox balance in the cell [172]. Consistent with this idea is the finding that peroxisomal proliferation seems to be regulated by the redox state of the organelle [19] and that abiotic stress such as salt stress induces the number of peroxisomes via Dnm1 and Vps1 [186]. 
The mechanisms of pexophagy in mammalian cells are only beginning to be unraveled [187, 188]. Here, selective autophagy of peroxisomes relies on cytosolic adaptor proteins such as p62 or NBR1, which recognize ubiquitinated cargo proteins and direct them to the autophagosome [189] (Figure 2). Pex5 is the peroxisomal receptor which upon ubiquitination triggers the process of pexophagy $[190,191]$. This process can be stimulated upon external oxidative stress or by the pharmacological induction of peroxisomal fatty acid oxidation and involves the ATM kinase [191, 192]. Activated ATM inhibits signaling through the mammalian TOR pathway and marks Pex5 for pexophagy by phosphorylation [193]. Subsequently, Pex5 is ubiquitinated, and p62dependent autophagy is initiated [191]. An E3 ubiquitin ligase responsible for Pex 5 ubiquitination has been recently found with Pex2 at least upon nutrient starvation conditions [194]. Taken together, these findings suggest that an excess of ROS produced at peroxisomes triggers the process of specific pexophagy to maintain a healthy redox balance in mammalian cells.

The removal of excess or damaged mitochondria by selective autophagy or mitophagy is an essential cellular homeostasis mechanism. In yeast, this process has been heavily studied upon nutrient starvation conditions, for example, upon depletion of a $\mathrm{N}$-source or in the stationary growth phase. These approaches have revealed many molecular insights of mitophagy; however, it remains unclear whether mitophagy in yeast actually degrades dysfunctional organelles [195]. Of central importance is the mitochondrial mitophagy receptor protein Atg32, which is localized at the outer mitochondrial membrane [196, 197]. Upon induction of mitophagy by starvation, Atg32 is phosphorylated by casein kinase 2 (CK2) at a specific serine (S114) residue [198]. Phosphorylation of Atg32 triggers its interaction with the cytosolic Atg11 adaptor protein. Therefore, this step is considered the initial signal in the targeting of mitochondrial to the preautophagosomal structure. Interestingly, there are at least two additional kinases which have been reported to be necessary for Atg32 phosphorylation, the Hog1 and Slt2 MAP kinases [199, 200]. Although both kinases cannot directly phosphorylate, the Atg32 receptor, the involvement of both kinases in stress signaling, could indicate a possible regulation of mitophagy by cellular stress stimuli additionally to nutrient shortage. Although still a matter of debate, there are experimental indications which suggest that mitophagy in yeast depends on mitochondrial fission. Generally, the mitochondrial fission proteins Fis1, Dnm1, Mdv1, and Caf4 seem to require for efficient mitophagy $[157,158,201]$. Specifically, during mitophagy, Atg11 directly targets the mitochondrial Dnm1 fission protein [158]. This suggests that mitophagy takes place close to the mitochondrial fission apparatus which would be necessary to separate the mitophagic cargo from the rest of the mitochondrial network [157]. Additional insights into the specific origins of mitophagy have been recently revealed by the finding that specific contact sites between the ER and mitochondria, the so called ERMES (ER mitochondria encounter structure), are necessary for mitophagy [202]. Thus, the initiation of mitophagy might require a close contact between the mitochondrial fission apparatus and the ER at strategic sites. It is less understood whether mitophagy in yeast is necessary to avoid cellular damage by dysfunctional mitochondria or whether it is activated upon oxidative stress. However, it has been reported that autophagic removal of excess mitochondria upon nutrient starvation is required to decrease intracellular ROS levels [203].

In mammalian cells, mitophagy has been intensively studied upon conditions which damage mitochondria by counteracting and eliminating their normal membrane potential [204] (Figure 2). In fact, loss of the mitochondrial membrane potential is one of the main triggers of mitophagy in mammalian models. Modulation of the mitochondrial shape is another important step in mitophagy, and inhibition of mitochondrial fission impairs the sequestration of damaged mitochondria by autophagosomes $[205,206]$. This suggests that fission of the organelle, which is often induced by cellular stress, is an important mechanism to segregate and eliminate damaged mitochondria from the otherwise healthy network. Failure in the execution of mitophagy surveillance pathways clearly impairs the cellular homeostasis. This has been demonstrated for the key regulators of mitophagy, PINK1 (PTEN-induced kinase), and Parkin, whose mutation can cause Parkinson's disease [207, 208]. PINK1 is a mitochondrial serine/threonine kinase encoded by the PARK6 locus [208], while Parkin is a cytosolic E3 ubiquitin ligase encoded by PARK2 [209]. Both proteins function in the same mitophagic pathway with PINK1 acting upstream of Parkin [210, 211]. Upon normal cell homeostasis, PINK1 is imported into mitochondria dependent on the mitochondrial membrane potential. In healthy mitochondria, it is cleaved by PARL at the inner mitochondrial membrane, thereby restricting its activity [212-214]. Loss of the mitochondrial membrane potential inhibits PINK1 import and provokes its accumulation at the outer mitochondrial membrane, where it recruits Parkin [215-217]. This is considered the key step to mark dysfunctional mitochondria for selective degradation. Once exposed at the mitochondrial surface, PINK1 forms larger aggregates, which stimulate PINK1 activity by autophosphorylation [218, 219]. Subsequently, the mitophagic pathway is initiated by PINK1 phosphorylation of both Parkin and ubiquitin in a positive feedback regulation. The phosphorylation of Parkin by PINK1 is necessary for its mitochondrial targeting and for its E3 ubiquitin ligase activity [215, 216, 220-223]. Furthermore, PINK1 phosphorylates ubiquitin, and phosphorylated ubiquitin seems to be the true targeting signal for Parkin [224-226]. Indeed, it has been recently revealed that Parkin accumulation at damaged mitochondria requires phosphorylated ubiquitin chains at the mitochondrial surface [227-229]. Ubiquitination of outer mitochondrial membrane proteins, most importantly of the mitofusins $\mathrm{Mfn} 1 / 2$, finally triggers the physical segregation of the damaged mitochondrial parts and degradation via autophagosomes [230-232]. The nature of the autophagy receptors which would recognize ubiquitinated damaged mitochondria has been controversial. However, a recent study discards the pexophagy receptors p62 or NBR1 and specifically implies Optineurin and NDP52 in PINK1/Parkin-mediated mitophagy [233]. 
Thus, phospho-ubiquitin at the surface of damaged mitochondria is the signal to recruit the NDP52 and Optineurin receptors, which in turn contact with components of the autophagy pathway such as ULK1 or LC3 to engage in the autophagic degradation of the dysfunctional mitochondria [234, 235].

It is important to note that mammalian cells apparently use different mitophagy pathways upon different stimuli. It has been demonstrated in mouse and human cells that oxidative stress is a sensitive mitophagy trigger, which reinforces the idea that the autophagic removal of damaged mitochondria is a physiologically relevant mechanism of cell homeostasis [236]. Apart from the PINK1/Parkin system, which responds to a loss of mitochondrial membrane potential, the Bcl2-L-13 protein (yeast Atg32 ortholog), the NIX/ BNIP3L, and the FUNDC1 mitochondrial outer membrane proteins have been involved in different types of mitophagy. Bcl2-L-13 seems to mediate mitophagy and mitochondrial fragmentation upon ETC damage [237], NIX/BNIP3L is involved in mitochondrial clearance during erythrocyte differentiation [238, 239], while FUNDC1 responds to hypoxic conditions [240]. It remains unclear to what degree these different mitophagy receptors act separately in the process of mitochondrial homeostasis.

\section{Physiological and Physical Interaction of Peroxisomes and Mitochondria}

In mammalian cells, peroxisomes and mitochondria functionally cooperate in the oxidative degradation of fatty acids by $\beta$-oxidation (see Figure 1). Both organelles have different but partially overlapping substrate specificities with regard to the chemical structure of the fatty acid to be metabolized. Therefore, peroxisomes and mitochondria cooperate in the homeostasis of lipids. The details of this metabolic cooperation have been summarized in excellent recent reviews $[241,242]$. It is important to note that both organelles share metabolic pathways apart from $\beta$-oxidation, for example in the detoxification of glyoxylate or in the degradation of special fatty acids via $\alpha$-oxidation [241, 243]. Also, it can be assumed that extensive peroxisomal $\alpha$-oxidation depends on the ATP supply from the mitochondrial respiratory chain. More importantly, it has been recently shown that there is an intimate crosstalk between peroxisomes and mitochondria in the homeostasis of intracellular ROS beyond the fact that both organelles are important sources and sinks for ROS as detailed above. It had been known that weakening the peroxisomal catalase activity either by pharmacological treatment or caused by the decreased protein import capacity triggered elevated oxidative stress and reduced enzyme activity at mitochondria $[104,107,244]$. The use of peroxisomally directed inducers of oxidative stress has then revealed that mitochondria indeed are downstream of peroxisomally generated ROS [101], which is manifested by mitochondrial fragmentation and cell death $[105,106]$. It has been suggested that mitochondrial oxidative damage is caused by lipid peroxidation originated in peroxisomes arguing against a simple diffusion of peroxisomal ROS to mitochondria. The mechanisms of the peroxisome-mitochondria ROS interplay

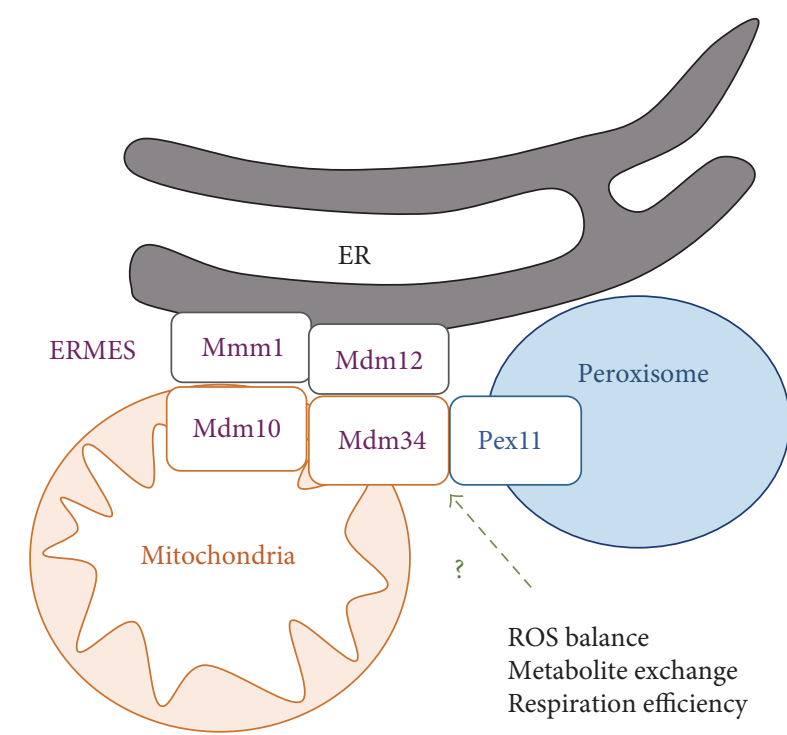

FIgURE 3: Overview of the yeast peroxisome-mitochondria contact site. The ERMES (endoplasmic reticulum mitochondria encounter structure) tethers mitochondria to the ER, but additionally establishes a contact between mitochondria and peroxisomes through the Mdm34-Pex11 interaction. The mitochondriaperoxisome tether might have dynamic functions in the regulation of ROS homeostasis, metabolite exchange between the two organelles, or the modulation of respiratory efficiency.

are currently unknown; however, interorganelle contact sites could explain this much more intimate relation of peroxisomes and mitochondria.

In the yeast model, the functional and physical interactions of peroxisomes with mitochondria have been studied intensively in recent years. As outlined above, fatty acid $\beta$ oxidation occurs exclusively in peroxisomes of budding yeast. Therefore, acetyl-CoA from peroxisomes has to be channeled into mitochondria via the carnitine shuttle and thus a direct contact of both organelles should favor this exchange of metabolites. Indeed, it has been shown that yeast peroxisomes preferentially localize close to specific mitochondrial sites which also contain contacts to the ER [245]. Recently, a possible physical tether between peroxisomes and mitochondria has been suggested by the interaction of the Pex11 protein involved in the biogenesis of the organelle with the mitochondrial Mdm34 protein [246] (Figure 3). Since Mdm34 is one of the structural components of the mitochondria-ER tether ERMES [247], this finding opens up the possibility that at least in yeast peroxisomes and mitochondria are physically connected at specific contact sites with the ER [248]. Recent evidence suggests that peroxisome-mitochondria contact sites might also exist in mammalian cells [249]. Since peroxisomes and mitochondria share components of their fission machineries and the fission of both organelles is important for their autophagic turnover, it is possible that this three-way contact is structurally important for the regulated turnover and therefore the ROS homeostasis of both organelles. Additionally, the mitochondria peroxisome interaction might be regulated dynamically to modulate ROS homeostasis, the interchange of metabolites or the respiratory efficiency. In this respect, it has been 
recently shown that the number of peroxisomes attached to the mitochondrial network increases upon cellular stress such as high salinity, which is known to also increase cellular ROS levels [186].

\section{Conclusions}

We know that peroxisomes and mitochondria are the crucial organelles in the homeostasis of eukaryotic ROS levels. Peroxisomes have traditionally lagged behind the importance of mitochondria in the production and detoxification of intracellular oxidative stress; however, this difference has largely disappeared in the past years. Current investigation is now deciphering the functional interplay of both organelles in the propagation and avoidance of oxidative damage in the cell. Peroxisomal and mitochondrial oxidative stress are intimately linked. Moreover, the dynamic fission of both organelles shares structural components, and the turnover of damaged peroxisomes and mitochondria as a mechanism to reduce intracellular oxidative stress will still be an important field of investigation. From a structural viewpoint, we are only beginning to understand how molecular tethers between peroxisomes and mitochondria, most likely via the ER, can contribute to interorganelle communication and coordinated quality control of both organelles. Therefore, investigation of the peroxisome-mitochondria connection and its regulation by stress will continue to contribute essential knowledge of how the redox homeostasis is maintained in young, environmentally challenged and aged eukaryotic cells.

\section{Conflicts of Interest}

The authors declare no conflict of interests.

\section{Acknowledgments}

Work from the authors' laboratory was supported by grants from Ministerio de Economía, Industria y Competitividad (BFU2016-75792-R) and from Ministerio de Economía y Competitividad (BFU2011-23326).

\section{References}

[1] D. F. Dai, Y. A. Chiao, D. J. Marcinek, H. H. Szeto, and P. S. Rabinovitch, "Mitochondrial oxidative stress in aging and healthspan," Longevity \& Healthspan, vol. 3, p. 6, 2014.

[2] M. Schrader, J. Costello, L. F. Godinho, and M. Islinger, "Peroxisome-mitochondria interplay and disease," Journal of Inherited Metabolic Disease, vol. 38, no. 4, pp. 681-702, 2015.

[3] C. M. Cipolla and I. J. Lodhi, "Peroxisomal dysfunction in age-related diseases," Trends in Endocrinology and Metabolism, vol. 28, no. 4, pp. 297-308, 2017.

[4] C. D. Duve and P. Baudhuin, "Peroxisomes (microbodies and related particles)," Physiological Reviews, vol. 46, no. 2, pp. 323-357, 1966.

[5] M. Fransen, M. Nordgren, B. Wang, and O. Apanasets, "Role of peroxisomes in ROS/RNS-metabolism: implications for human disease," Biochimica et Biophysica Acta
(BBA) - Molecular Basis of Disease, vol. 1822, no. 9, pp. 1363-1373, 2012.

[6] M. Nordgren and M. Fransen, "Peroxisomal metabolism and oxidative stress,” Biochimie, vol. 98, pp. 56-62, 2014.

[7] J. J. Smith and J. D. Aitchison, "Peroxisomes take shape," Nature Reviews. Molecular Cell Biology, vol. 14, no. 12, pp. 803-817, 2013.

[8] A. Boveris, N. Oshino, and B. Chance, "The cellular production of hydrogen peroxide," The Biochemical Journal, vol. 128, no. 3, pp. 617-630, 1972.

[9] I. J. Lodhi and C. F. Semenkovich, "Peroxisomes: a nexus for lipid metabolism and cellular signaling," Cell Metabolism, vol. 19, no. 3, pp. 380-392, 2014.

[10] M. Schrader, S. Grille, H. D. Fahimi, and M. Islinger, "Peroxisome interactions and cross-talk with other subcellular compartments in animal cells," Sub-Cellular Biochemistry, vol. 69, pp. 1-22, 2013.

[11] J. J. Smith and J. D. Aitchison, "Regulation of peroxisome dynamics," Current Opinion in Cell Biology, vol. 21, no. 1, pp. 119-126, 2009.

[12] S. Kumar, A. Kawalek, and I. J. v. d. Klei, "Peroxisomal quality control mechanisms," Current Opinion in Microbiology, vol. 22, pp. 30-37, 2014.

[13] C. W. v. Roermund, H. R. Waterham, L. Ijlst, and R. J. Wanders, "Fatty acid metabolism in Saccharomyces cerevisiae," Cellular and Molecular Life Sciences, vol. 60, no. 9, pp. 1838-1851, 2003.

[14] J. K. Reddy and T. Hashimoto, "Peroxisomal beta-oxidation and peroxisome proliferator-activated receptor alpha: an adaptive metabolic system," Annual Review of Nutrition, vol. 21, pp. 193-230, 2001.

[15] V. D. Antonenkov, S. Grunau, S. Ohlmeier, and J. K. Hiltunen, "Peroxisomes are oxidative organelles," Antioxidants \& Redox Signaling, vol. 13, no. 4, pp. 525-537, 2010.

[16] W. Hansberg, R. Salas-Lizana, and L. Dominguez, "Fungal catalases: function, phylogenetic origin and structure," Archives of Biochemistry and Biophysics, vol. 525, no. 2, pp. 170-180, 2012.

[17] H. N. Kirkman and G. F. Gaetani, "Mammalian catalase: a venerable enzyme with new mysteries," Trends in Biochemical Sciences, vol. 32, no. 1, pp. 44-50, 2007.

[18] A. Kawalek, S. D. Lefevre, M. Veenhuis, and I. J. v. d. Klei, "Peroxisomal catalase deficiency modulates yeast lifespan depending on growth conditions," Aging (Albany NY), vol. 5, no. 1, pp. 67-83, 2013.

[19] T. Ohdate and Y. Inoue, "Involvement of glutathione peroxidase 1 in growth and peroxisome formation in Saccharomyces cerevisiae in oleic acid medium," Biochimica et Biophysica Acta (BBA) - Molecular and Cell Biology of Lipids, vol. 1821, no. 9, pp. 1295-1305, 2012.

[20] H. Kasai, Y. Okada, S. Nishimura, M. S. Rao, and J. K. Reddy, "Formation of 8-hydroxydeoxyguanosine in liver DNA of rats following long-term exposure to a peroxisome proliferator," Cancer Research, vol. 49, no. 10, pp. 26032605, 1989.

[21] M. L. O'Brien, T. P. Twaroski, M. L. Cunningham, H. P. Glauert, and B. T. Spear, "Effects of peroxisome proliferators on antioxidant enzymes and antioxidant vitamins in rats and hamsters," Toxicological Sciences, vol. 60, no. 2, pp. 271-278, 2001. 
[22] J. K. Reddy, S. K. Goel, M. R. Nemali et al., "Transcription regulation of peroxisomal fatty acyl-CoA oxidase and enoylCoA hydratase/3-hydroxyacyl-CoA dehydrogenase in rat liver by peroxisome proliferators," Proceedings of the National Academy of Sciences of the United States of America, vol. 83, no. 6, pp. 1747-1751, 1986.

[23] S. Diano, Z. W. Liu, J. K. Jeong et al., "Peroxisome proliferation-associated control of reactive oxygen species sets melanocortin tone and feeding in diet-induced obesity," Nature Medicine, vol. 17, no. 9, pp. 1121-1127, 2011.

[24] L. Hulshagen, O. Krysko, A. Bottelbergs et al., "Absence of functional peroxisomes from mouse CNS causes dysmyelination and axon degeneration," The Journal of Neuroscience, vol. 28, no. 15, pp. 4015-4027, 2008.

[25] O. Krysko, L. Hulshagen, A. Janssen et al., "Neocortical and cerebellar developmental abnormalities in conditions of selective elimination of peroxisomes from brain or from liver," Journal of Neuroscience Research, vol. 85, no. 1, pp. 58-72, 2007.

[26] L. Goth and J. W. Eaton, "Hereditary catalase deficiencies and increased risk of diabetes," Lancet, vol. 356, no. 9244, pp. 1820-1821, 2000.

[27] O. I. Petriv and R. A. Rachubinski, "Lack of peroxisomal catalase causes a progeric phenotype in Caenorhabditis elegans," The Journal of Biological Chemistry, vol. 279, no. 19, pp. 19996-20001, 2004.

[28] N. A. Bonekamp, A. Volkl, H. D. Fahimi, and M. Schrader, "Reactive oxygen species and peroxisomes: struggling for balance," BioFactors, vol. 35, no. 4, pp. 346-355, 2009.

[29] M. Schrader and H. D. Fahimi, "Peroxisomes and oxidative stress," Biochimica et Biophysica Acta (BBA) - Molecular Cell Research, vol. 1763, no. 12, pp. 1755-1766, 2006.

[30] H. M. McBride, M. Neuspiel, and S. Wasiak, "Mitochondria: more than just a powerhouse," Current Biology, vol. 16, no. 14, pp. R551-R560, 2006.

[31] J. Nunnari and A. Suomalainen, "Mitochondria: in sickness and in health," Cell, vol. 148, no. 6, pp. 1145-1159, 2012.

[32] S. W. Tait and D. R. Green, "Mitochondrial regulation of cell death," Cold Spring Harbor Perspectives in Biology, vol. 5, no. 9, 2013.

[33] H. Vakifahmetoglu-Norberg, A. T. Ouchida, and E. Norberg, "The role of mitochondria in metabolism and cell death," Biochemical and Biophysical Research Communications, vol. 482, no. 3, pp. 426-431, 2017.

[34] J. F. Turrens, "Mitochondrial formation of reactive oxygen species," The Journal of Physiology, vol. 552, Part 2, pp. 335-344, 2003.

[35] J. Cadet and J. R. Wagner, "Oxidatively generated base damage to cellular DNA by hydroxyl radical and one-electron oxidants: similarities and differences," Archives of Biochemistry and Biophysics, vol. 557, pp. 47-54, 2014.

[36] A. Weidinger and A. V. Kozlov, "Biological activities of reactive oxygen and nitrogen species: oxidative stress versus signal transduction," Biomolecules, vol. 5, no. 2, pp. 472484, 2015.

[37] M. P. Murphy, "How mitochondria produce reactive oxygen species," The Biochemical Journal, vol. 417, no. 1, pp. 1-13, 2009.

[38] J. Ruszkiewicz and J. Albrecht, "Changes in the mitochondrial antioxidant systems in neurodegenerative diseases and acute brain disorders," Neurochemistry International, vol. 88, pp. 66-72, 2015.

[39] A. A. Starkov, "The role of mitochondria in reactive oxygen species metabolism and signaling," Annals of the New York Academy of Sciences, vol. 1147, pp. 37-52, 2008.

[40] A. Y. Andreyev, Y. E. Kushnareva, A. N. Murphy, and A. A. Starkov, "Mitochondrial ROS metabolism: 10 years later," Biochemistry (Mosc), vol. 80, no. 5, pp. 517-531, 2015.

[41] A. Y. Andreyev, Y. E. Kushnareva, and A. A. Starkov, "Mitochondrial metabolism of reactive oxygen species," Biochemistry (Mosc), vol. 70, no. 2, pp. 200-214, 2005.

[42] A. Okado-Matsumoto and I. Fridovich, "Subcellular distribution of superoxide dismutases (SOD) in rat liver: $\mathrm{Cu}, \mathrm{Zn}-\mathrm{SOD}$ in mitochondria," The Journal of Biological Chemistry, vol. 276, no. 42, pp. 38388-38393, 2001.

[43] R. Brigelius-Flohe and M. Maiorino, "Glutathione peroxidases," Biochimica et Biophysica Acta (BBA) - General Subjects, vol. 1830, no. 5, pp. 3289-3303, 2013.

[44] H. Imai and Y. Nakagawa, "Biological significance of phospholipid hydroperoxide glutathione peroxidase (PHGPx, GPx4) in mammalian cells," Free Radical Biology and Medicine, vol. 34, no. 2, pp. 145-169, 2003.

[45] C. Johansson, C. H. Lillig, and A. Holmgren, "Human mitochondrial glutaredoxin reduces S-glutathionylated proteins with high affinity accepting electrons from either glutathione or thioredoxin reductase," The Journal of Biological Chemistry, vol. 279, no. 9, pp. 7537-7543, 2004.

[46] I. Banmeyer, C. Marchand, A. Clippe, and B. Knoops, "Human mitochondrial peroxiredoxin 5 protects from mitochondrial DNA damages induced by hydrogen peroxide," FEBS Letters, vol. 579, no. 11, pp. 2327-2333, 2005.

[47] Z. Cao, J. G. Lindsay, and N. W. Isaacs, "Mitochondrial peroxiredoxins," Sub-Cellular Biochemistry, vol. 44, pp. 295315, 2007.

[48] T. S. Chang, C. S. Cho, S. Park, S. Yu, S. W. Kang, and S. G. Rhee, "Peroxiredoxin III, a mitochondrion-specific peroxidase, regulates apoptotic signaling by mitochondria," The Journal of Biological Chemistry, vol. 279, no. 40, pp. 4197541984, 2004.

[49] E. M. Hanschmann, J. R. Godoy, C. Berndt, C. Hudemann, and C. H. Lillig, "Thioredoxins, glutaredoxins, and peroxiredoxins-molecular mechanisms and health significance: from cofactors to antioxidants to redox signaling," Antioxidants \& Redox Signaling, vol. 19, no. 13, pp. 1539-1605, 2013.

[50] D. Silva-Adaya, M. E. Gonsebatt, and J. Guevara, "Thioredoxin system regulation in the central nervous system: experimental models and clinical evidence," Oxidative Medicine and Cellular Longevity, vol. 2014, Article ID 590808, 13 pages, 2014.

[51] A. M. James, R. A. Smith, and M. P. Murphy, "Antioxidant and prooxidant properties of mitochondrial coenzyme Q," Archives of Biochemistry and Biophysics, vol. 423, no. 1, pp. 47-56, 2004.

[52] M. Kirsch and H. D. Groot, "NAD $(\mathrm{P}) \mathrm{H}$, a directly operating antioxidant?" The FASEB Journal, vol. 15, no. 9, pp. 15691574, 2001.

[53] G. T. Vatassery, W. E. Smith, H. T. Quach, and J. C. Lai, "In vitro oxidation of vitamin $\mathrm{E}$, vitamin $\mathrm{C}$, thiols and cholesterol in rat brain mitochondria incubated with free 
radicals," Neurochemistry International, vol. 26, no. 5, pp. 527-535, 1995.

[54] M. B. Toledano, A. Delaunay-Moisan, C. E. Outten, and A. Igbaria, "Functions and cellular compartmentation of the thioredoxin and glutathione pathways in yeast," Antioxidants \& Redox Signaling, vol. 18, no. 13, pp. 1699-1711, 2013.

[55] S. D. Ravindranath and I. Fridovich, "Isolation and characterization of a manganese-containing superoxide dismutase from yeast," The Journal of Biological Chemistry, vol. 250, no. 15 , pp. 6107-6112, 1975.

[56] L. A. Sturtz, K. Diekert, L. T. Jensen, R. Lill, and V. C. Culotta, "A fraction of yeast $\mathrm{Cu}, \mathrm{Zn}$-superoxide dismutase and its metallochaperone, CCS, localize to the intermembrane space of mitochondria. A physiological role for SOD1 in guarding against mitochondrial oxidative damage," The Journal of Biological Chemistry, vol. 276, no. 41, pp. 38084-38089, 2001.

[57] V. Y. Petrova, D. Drescher, A. V. Kujumdzieva, and M. J. Schmitt, "Dual targeting of yeast catalase A to peroxisomes and mitochondria," The Biochemical Journal, vol. 380, Part 2, pp. 393-400, 2004.

[58] D. Harman, "Aging: a theory based on free radical and radiation chemistry," Journal of Gerontology, vol. 11, no. 3, pp. 298-300, 1956.

[59] R. J. Mockett, W. C. Orr, J. J. Rahmandar et al., "Overexpression of Mn-containing superoxide dismutase in transgenic Drosophila melanogaster," Archives of Biochemistry and Biophysics, vol. 371, no. 2, pp. 260-269, 1999.

[60] W. C. Orr and R. S. Sohal, "Does overexpression of $\mathrm{Cu}, \mathrm{Zn}$-SOD extend life span in Drosophila melanogaster?" Experimental Gerontology, vol. 38, no. 3, pp. 227-230, 2003.

[61] T. T. Huang, E. J. Carlson, A. M. Gillespie, Y. Shi, and C. J. Epstein, "Ubiquitous overexpression of CuZn superoxide dismutase does not extend life span in mice," The Journals of Gerontology. Series A, Biological Sciences and Medical Sciences, vol. 55, no. 1, pp. B5-B9, 2000.

[62] Y. C. Jang, V. I. Perez, W. Song et al., "Overexpression of Mn superoxide dismutase does not increase life span in mice," The Journals of Gerontology. Series A, Biological Sciences and Medical Sciences, vol. 64, no. 11, pp. 1114-1125, 2009.

[63] V. I. Perez, H. V. Remmen, A. Bokov, C. J. Epstein, J. Vijg, and A. Richardson, "The overexpression of major antioxidant enzymes does not extend the lifespan of mice," Aging Cell, vol. 8, no. 1, pp. 73-75, 2009.

[64] G. Bjelakovic, D. Nikolova, L. L. Gluud, R. G. Simonetti, and C. Gluud, "Mortality in randomized trials of antioxidant supplements for primary and secondary prevention: systematic review and meta-analysis," The Journal of the American Medical Association, vol. 297, no. 8, pp. 842-857, 2007.

[65] D. Harman, “The biologic clock: the mitochondria?” Journal of the American Geriatrics Society, vol. 20, no. 4, pp. 145-147, 1972.

[66] R. S. Balaban, S. Nemoto, and T. Finkel, "Mitochondria, oxidants, and aging," Cell, vol. 120, no. 4, pp. 483-495, 2005.

[67] A. V. Kudryavtseva, G. S. Krasnov, A. A. Dmitriev et al., "Mitochondrial dysfunction and oxidative stress in aging and cancer," Oncotarget, vol. 7, no. 29, pp. 44879-44905, 2016.

[68] A. O. Oyewole and M. A. Birch-Machin, "Mitochondriatargeted antioxidants," The FASEB Journal, vol. 29, no. 12, pp. 4766-4771, 2015.
[69] S. E. Schriner, N. J. Linford, G. M. Martin et al., "Extension of murine life span by overexpression of catalase targeted to mitochondria," Science, vol. 308 , no. 5730, pp. 19091911, 2005.

[70] V. N. Anisimov, M. V. Egorov, M. S. Krasilshchikova et al., "Effects of the mitochondria-targeted antioxidant SkQ1 on lifespan of rodents," Aging (Albany NY), vol. 3, no. 11, pp. 1110-1119, 2011.

[71] A. Trifunovic, A. Wredenberg, M. Falkenberg et al., "Premature ageing in mice expressing defective mitochondrial DNA polymerase," Nature, vol. 429, no. 6990, pp. 417-423, 2004.

[72] G. C. Kujoth, A. Hiona, T. D. Pugh et al., "Mitochondrial DNA mutations, oxidative stress, and apoptosis in mammalian aging," Science, vol. 309, no. 5733, pp. 481-484, 2005.

[73] D. F. Dai, T. Chen, J. Wanagat et al., “Age-dependent cardiomyopathy in mitochondrial mutator mice is attenuated by overexpression of catalase targeted to mitochondria," Aging Cell, vol. 9, no. 4, pp. 536-544, 2010.

[74] K. Kirby, J. Hu, A. J. Hilliker, and J. P. Phillips, "RNA interference-mediated silencing of Sod2 in Drosophila leads to early adult-onset mortality and elevated endogenous oxidative stress," Proceedings of the National Academy of Sciences of the United States of America, vol. 99, no. 25, pp. 16162-16167, 2002.

[75] A. Duttaroy, A. Paul, M. Kundu, and A. Belton, "A Sod2 null mutation confers severely reduced adult life span in Drosophila," Genetics, vol. 165, no. 4, pp. 2295-2299, 2003.

[76] A. Paul, A. Belton, S. Nag, I. Martin, M. S. Grotewiel, and A. Duttaroy, "Reduced mitochondrial SOD displays mortality characteristics reminiscent of natural aging," Mechanisms of Ageing and Development, vol. 128, no. 11-12, pp. 706-716, 2007.

[77] V. D. Longo and P. Fabrizio, "Chronological aging in Saccharomyces cerevisiae," Sub-Cellular Biochemistry, vol. 57, pp. 101-121, 2012.

[78] V. D. Longo, G. S. Shadel, M. Kaeberlein, and B. Kennedy, "Replicative and chronological aging in Saccharomyces cerevisiae," Cell Metabolism, vol. 16, no. 1, pp. 18-31, 2012.

[79] E. Parrella and V. D. Longo, "The chronological life span of Saccharomyces cerevisiae to study mitochondrial dysfunction and disease," Methods, vol. 46, no. 4, pp. 256-262, 2008.

[80] V. D. Longo, E. B. Gralla, and J. S. Valentine, "Superoxide dismutase activity is essential for stationary phase survival in Saccharomyces cerevisiae. Mitochondrial production of toxic oxygen species in vivo," The Journal of Biological Chemistry, vol. 271, no. 21, pp. 12275-12280, 1996.

[81] V. P. Skulachev, V. N. Anisimov, Y. N. Antonenko et al., "An attempt to prevent senescence: a mitochondrial approach," Biochimica et Biophysica Acta (BBA) - Bioenergetics, vol. 1787, no. 5, pp. 437-461, 2009.

[82] H. H. Szeto, "First-in-class cardiolipin-protective compound as a therapeutic agent to restore mitochondrial bioenergetics," British Journal of Pharmacology, vol. 171, no. 8, pp. 2029-2050, 2014.

[83] R. A. Smith, R. C. Hartley, H. M. Cocheme, and M. P. Murphy, "Mitochondrial pharmacology," Trends in Pharmacological Sciences, vol. 33, no. 6, pp. 341-352, 2012.

[84] B. A. Feniouk and V. P. Skulachev, "Cellular and molecular mechanisms of action of mitochondria-targeted antioxidants," Current Aging Science, vol. 10, no. 1, pp. 41-48, 2017. 
[85] R. B. Hamanaka and N. S. Chandel, "Mitochondrial reactive oxygen species regulate hypoxic signaling," Current Opinion in Cell Biology, vol. 21, no. 6, pp. 894-899, 2009.

[86] R. B. Hamanaka and N. S. Chandel, "Mitochondrial reactive oxygen species regulate cellular signaling and dictate biological outcomes," Trends in Biochemical Sciences, vol. 35, no. 9, pp. 505-513, 2010.

[87] C. Mammucari and R. Rizzuto, "Signaling pathways in mitochondrial dysfunction and aging," Mechanisms of Ageing and Development, vol. 131, no. 7-8, pp. 536-543, 2010.

[88] M. Ristow and K. Zarse, "How increased oxidative stress promotes longevity and metabolic health: the concept of mitochondrial hormesis (mitohormesis)," Experimental Gerontology, vol. 45, no. 6, pp. 410-418, 2010.

[89] J. Yun and T. Finkel, "Mitohormesis," Cell Metabolism, vol. 19, no. 5, pp. 757-766, 2014.

[90] M. Ristow and S. Schmeisser, "Extending life span by increasing oxidative stress," Free Radical Biology \& Medicine, vol. 51, no. 2, pp. 327-336, 2011.

[91] Y. Wang and S. Hekimi, "Mitochondrial dysfunction and longevity in animals: untangling the knot," Science, vol. 350, no. 6265, pp. 1204-1207, 2015.

[92] S. J. Lee, A. B. Hwang, and C. Kenyon, "Inhibition of respiration extends $C$. elegans life span via reactive oxygen species that increase HIF-1 activity," Current Biology, vol. 20, no. 23, pp. 2131-2136, 2010.

[93] B. M. Zid, A. N. Rogers, S. D. Katewa et al., “4E-BP extends lifespan upon dietary restriction by enhancing mitochondrial activity in Drosophila," Cell, vol. 139, no. 1, pp. 149-160, 2009.

[94] S. J. Lin, M. Kaeberlein, A. A. Andalis et al., "Calorie restriction extends Saccharomyces cerevisiae lifespan by increasing respiration," Nature, vol. 418, no. 6895, pp. 344348, 2002.

[95] T. J. Schulz, K. Zarse, A. Voigt, N. Urban, M. Birringer, and M. Ristow, "Glucose restriction extends Caenorhabditis elegans life span by inducing mitochondrial respiration and increasing oxidative stress," Cell Metabolism, vol. 6, no. 4, pp. 280-293, 2007.

[96] C. Skinner and S. J. Lin, "Effects of calorie restriction on life span of microorganisms," Applied Microbiology and Biotechnology, vol. 88, no. 4, pp. 817-828, 2010.

[97] J. R. Delaney, C. Murakami, A. Chou et al., "Dietary restriction and mitochondrial function link replicative and chronological aging in Saccharomyces cerevisiae," Experimental Gerontology, vol. 48, no. 10, pp. 1006-1013, 2013.

[98] P. Fabrizio, L. L. Liou, V. N. Moy et al., "SOD2 functions downstream of Sch9 to extend longevity in yeast," Genetics, vol. 163, no. 1, pp. 35-46, 2003.

[99] N. D. Bonawitz, M. Chatenay-Lapointe, Y. Pan, and G. S. Shadel, "Reduced TOR signaling extends chronological life span via increased respiration and upregulation of mitochondrial gene expression," Cell Metabolism, vol. 5, no. 4, pp. 265277, 2007.

[100] Y. Pan, E. A. Schroeder, A. Ocampo, A. Barrientos, and G. S. Shadel, "Regulation of yeast chronological life span by TORC1 via adaptive mitochondrial ROS signaling," Cell Metabolism, vol. 13, no. 6, pp. 668-678, 2011.

[101] M. Fransen, M. Nordgren, B. Wang, O. Apanasets, and P. P. $\mathrm{V}$. Veldhoven, "Aging, age-related diseases and peroxisomes,” Sub-Cellular Biochemistry, vol. 69, pp. 45-65, 2013.
[102] C. R. Giordano and S. R. Terlecky, "Peroxisomes, cell senescence, and rates of aging," Biochimica et Biophysica Acta (BBA) - Molecular Basis of Disease, vol. 1822, no. 9, pp. 1358-1362, 2012.

[103] I. Hwang, J. Lee, J. Y. Huh et al., "Catalase deficiency accelerates diabetic renal injury through peroxisomal dysfunction," Diabetes, vol. 61, no. 3, pp. 728-738, 2012.

[104] P. A. Walton and M. Pizzitelli, "Effects of peroxisomal catalase inhibition on mitochondrial function," Frontiers in Physiology, vol. 3, p. 108, 2012.

[105] B. Wang, P. P. V. Veldhoven, C. Brees et al., "Mitochondria are targets for peroxisome-derived oxidative stress in cultured mammalian cells," Free Radical Biology \& Medicine, vol. 65, pp. 882-894, 2013.

[106] O. Ivashchenko, P. P. V. Veldhoven, C. Brees, Y. S. Ho, S. R. Terlecky, and M. Fransen, "Intraperoxisomal redox balance in mammalian cells: oxidative stress and interorganellar cross-talk," Molecular Biology of the Cell, vol. 22, no. 9, pp. 1440-1451, 2011.

[107] J. E. Legakis, J. I. Koepke, C. Jedeszko et al., "Peroxisome senescence in human fibroblasts," Molecular Biology of the Cell, vol. 13, no. 12, pp. 4243-4255, 2002.

[108] V. Narayan, T. Ly, E. Pourkarimi et al., "Deep proteome analysis identifies age-related processes in C. elegans," Cell Systems, vol. 3, no. 2, pp. 144-159, 2016.

[109] C. Ma, D. Hagstrom, S. G. Polley, and S. Subramani, "Redoxregulated cargo binding and release by the peroxisomal targeting signal receptor, Pex5," The Journal of Biological Chemistry, vol. 288, no. 38, pp. 27220-27231, 2013.

[110] O. Apanasets, C. P. Grou, P. P. V. Veldhoven et al., "PEX5, the shuttling import receptor for peroxisomal matrix proteins, is a redox-sensitive protein," Traffic, vol. 15, no. 1, pp. 94-103, 2014.

[111] E. Bener Aksam, H. Jungwirth, S. D. Kohlwein et al., "Absence of the peroxiredoxin Pmp20 causes peroxisomal protein leakage and necrotic cell death," Free Radical Biology \& Medicine, vol. 45, no. 8, pp. 1115-1124, 2008.

[112] P. A. Marshall, J. M. Dyer, M. E. Quick, and J. M. Goodman, "Redox-sensitive homodimerization of Pex11p: a proposed mechanism to regulate peroxisomal division," The Journal of Cell Biology, vol. 135, no. 1, pp. 123-137, 1996.

[113] X. Wang, S. Li, Y. Liu, and C. Ma, "Redox regulated peroxisome homeostasis," Redox Biology, vol. 4, pp. 104-108, 2015.

[114] S. D. Lefevre, S. Kumar, and I. J. van del Klei, "Inhibition of peroxisome fission, but not mitochondrial fission, increases yeast chronological lifespan," Cell Cycle, vol. 14, no. 11, pp. 1698-1703, 2015.

[115] R. G. Allen, M. Tresini, B. P. Keogh, D. L. Doggett, and V. J. Cristofalo, "Differences in electron transport potential, antioxidant defenses, and oxidant generation in young and senescent fetal lung fibroblasts (WI-38)," Journal of Cellular Physiology, vol. 180, no. 1, pp. 114-122, 1999.

[116] E. Hutter, K. Renner, G. Pfister, P. Stockl, P. Jansen-Durr, and E. Gnaiger, "Senescence-associated changes in respiration and oxidative phosphorylation in primary human fibroblasts," The Biochemical Journal, vol. 380, Part 3, pp. 919928, 2004.

[117] O. Moiseeva, V. Bourdeau, A. Roux, X. Deschenes-Simard, and G. Ferbeyre, "Mitochondrial dysfunction contributes to oncogene-induced senescence," Molecular and Cellular Biology, vol. 29, no. 16, pp. 4495-4507, 2009. 
[118] J. F. Passos, G. Saretzki, S. Ahmed et al., "Mitochondrial dysfunction accounts for the stochastic heterogeneity in telomere-dependent senescence," PLoS Biology, vol. 5, no. 5, article e110, 2007.

[119] C. Lopez-Otin, M. A. Blasco, L. Partridge, M. Serrano, and G. Kroemer, "The hallmarks of aging," Cell, vol. 153, no. 6, pp. 1194-1217, 2013.

[120] T. Finkel, “The metabolic regulation of aging," Nature Medicine, vol. 21, no. 12, pp. 1416-1423, 2015.

[121] N. Sun, R. J. Youle, and T. Finkel, "The mitochondrial basis of aging," Molecular Cell, vol. 61, no. 5, pp. 654-666, 2016.

[122] C. Correia-Melo and J. F. Passos, "Mitochondria: Are they causal players in cellular senescence?" Biochimica et Biophysica Acta (BBA) - Bioenergetics, vol. 1847, no. 11, pp. 13731379, 2015.

[123] V. I. Korolchuk, S. Miwa, B. Carroll, and T. v. Zglinicki, "Mitochondria in cell senescence: Is mitophagy the weakest link?” eBioMedicine, vol. 21, pp. 7-13, 2017.

[124] A. Trifunovic and N. G. Larsson, "Mitochondrial dysfunction as a cause of ageing," Journal of Internal Medicine, vol. 263, no. 2, pp. 167-178, 2008.

[125] S. Judge, Y. M. Jang, A. Smith, T. Hagen, and C. Leeuwenburgh, "Age-associated increases in oxidative stress and antioxidant enzyme activities in cardiac interfibrillar mitochondria: implications for the mitochondrial theory of aging," The FASEB Journal, vol. 19, no. 3, pp. 419-421, 2005.

[126] R. Ventura-Clapier, A. Garnier, and V. Veksler, "Transcriptional control of mitochondrial biogenesis: the central role of PGC-1alpha," Cardiovascular Research, vol. 79, no. 2, pp. 208-217, 2008.

[127] R. Ventura-Clapier, A. Garnier, V. Veksler, and F. Joubert, "Bioenergetics of the failing heart," Biochimica et Biophysica Acta (BBA) - Molecular Cell Research, vol. 1813, no. 7, pp. 1360-1372, 2011.

[128] A. J. Murray, R. E. Anderson, G. C. Watson, G. K. Radda, and K. Clarke, "Uncoupling proteins in human heart," Lancet, vol. 364, no. 9447, pp. 1786-1788, 2004.

[129] S. Goffart, J. C. v. Kleist-Retzow, and R. J. Wiesner, "Regulation of mitochondrial proliferation in the heart: power-plant failure contributes to cardiac failure in hypertrophy," Cardiovascular Research, vol. 64, no. 2, pp. 198-207, 2004.

[130] S. DiMauro and E. A. Schon, "Mitochondrial respiratorychain diseases," The New England Journal of Medicine, vol. 348, no. 26, pp. 2656-2668, 2003.

[131] L. Dupuis, "Mitochondrial quality control in neurodegenerative diseases," Biochimie, vol. 100, pp. 177-183, 2014.

[132] J. Gao, L. Wang, J. Liu, F. Xie, B. Su, and X. Wang, “Abnormalities of mitochondrial dynamics in neurodegenerative diseases," Antioxidants (Basel), vol. 6, no. 2, 2017.

[133] M. J. Keogh and P. F. Chinnery, "Mitochondrial DNA mutations in neurodegeneration," Biochimica et Biophysica Acta (BBA) - Bioenergetics, vol. 1847, no. 11, pp. 1401-11, 2015.

[134] B. Terni, J. Boada, M. Portero-Otin, R. Pamplona, and I. Ferrer, "Mitochondrial ATP-synthase in the entorhinal cortex is a target of oxidative stress at stages I/II of Alzheimer's disease pathology," Brain Pathology, vol. 20, no. 1, pp. 222-233, 2010.

[135] J. W. Lustbader, M. Cirilli, C. Lin et al., "ABAD directly links Abeta to mitochondrial toxicity in Alzheimer's disease," Science, vol. 304, no. 5669, pp. 448-452, 2004.
[136] P. J. Crouch, R. Blake, J. A. Duce et al., "Copper-dependent inhibition of human cytochrome c oxidase by a dimeric conformer of amyloid-beta1-42," The Journal of Neuroscience, vol. 25, no. 3, pp. 672-679, 2005.

[137] H. Atamna and K. Boyle, "Amyloid-beta peptide binds with heme to form a peroxidase: relationship to the cytopathologies of Alzheimer's disease," Proceedings of the National Academy of Sciences of the United States of America, vol. 103, no. 9, pp. 3381-3386, 2006.

[138] E. Ferreiro, C. R. Oliveira, and C. M. Pereira, "The release of calcium from the endoplasmic reticulum induced by amyloid-beta and prion peptides activates the mitochondrial apoptotic pathway," Neurobiology of Disease, vol. 30, no. 3, pp. 331-342, 2008.

[139] P. E. Coskun, M. F. Beal, and D. C. Wallace, "Alzheimer's brains harbor somatic mtDNA control-region mutations that suppress mitochondrial transcription and replication," Proceedings of the National Academy of Sciences of the United States of America, vol. 101, no. 29, pp. 1072610731, 2004.

[140] D. H. Cho, T. Nakamura, J. Fang et al., "S-nitrosylation of Drp1 mediates beta-amyloid-related mitochondrial fission and neuronal injury," Science, vol. 324, no. 5923, pp. 102105, 2009.

[141] M. Manczak, M. J. Calkins, and P. H. Reddy, "Impaired mitochondrial dynamics and abnormal interaction of amyloid beta with mitochondrial protein Drp1 in neurons from patients with Alzheimer's disease: implications for neuronal damage," Human Molecular Genetics, vol. 20, no. 13, pp. 2495-2509, 2011.

[142] B. Thomas and M. F. Beal, "Parkinson's disease," Human Molecular Genetics, vol. 16, no. 2, pp. R183-R194, 2007.

[143] F. Kamp, N. Exner, A. K. Lutz et al., "Inhibition of mitochondrial fusion by alpha-synuclein is rescued by PINK1, Parkin and DJ-1," The EMBO Journal, vol. 29, no. 20, pp. 35713589, 2010.

[144] M. K. McCoy and M. R. Cookson, "DJ-1 regulation of mitochondrial function and autophagy through oxidative stress," Autophagy, vol. 7, no. 5, pp. 531-532, 2011.

[145] X. Wang, T. G. Petrie, Y. Liu, J. Liu, H. Fujioka, and X. Zhu, "Parkinson's disease-associated DJ-1 mutations impair mitochondrial dynamics and cause mitochondrial dysfunction," Journal of Neurochemistry, vol. 121, no. 5, pp. 830-839, 2012.

[146] H. Mortiboys, K. K. Johansen, J. O. Aasly, and O. Bandmann, "Mitochondrial impairment in patients with Parkinson's disease with the G2019S mutation in LRRK2," Neurology, vol. 75, no. 22, pp. 2017-2020, 2010.

[147] X. Wang, M. H. Yan, H. Fujioka et al., "LRRK2 regulates mitochondrial dynamics and function through direct interaction with DLP1," Human Molecular Genetics, vol. 21, no. 9, pp. 1931-1944, 2012.

[148] A. M. Pickrell and R. J. Youle, "The roles of PINK1, parkin, and mitochondrial fidelity in Parkinson's disease," Neuron, vol. 85, no. 2, pp. 257-273, 2015.

[149] D. P. Narendra and R. J. Youle, “Targeting mitochondrial dysfunction: role for PINK1 and Parkin in mitochondrial quality control," Antioxidants \& Redox Signaling, vol. 14, no. 10, pp. 1929-1938, 2011.

[150] D. C. Chan, "Fusion and fission: interlinked processes critical for mitochondrial health," Annual Review of Genetics, vol. 46, pp. 265-287, 2012. 
[151] S. A. Detmer and D. C. Chan, "Functions and dysfunctions of mitochondrial dynamics," Nature Reviews. Molecular Cell Biology, vol. 8, no. 11, pp. 870-879, 2007.

[152] H. Chen and D. C. Chan, "Mitochondrial dynamics-fusion, fission, movement, and mitophagy-in neurodegenerative diseases," Human Molecular Genetics, vol. 18, no. R2, pp. R169-R176, 2009.

[153] Y. S. Yoon, D. S. Yoon, I. K. Lim et al., "Formation of elongated giant mitochondria in DFO-induced cellular senescence: involvement of enhanced fusion process through modulation of Fis1," Journal of Cellular Physiology, vol. 209, no. 2, pp. 468-480, 2006.

[154] S. Lee, S. Y. Jeong, W. C. Lim et al., "Mitochondrial fission and fusion mediators, hFis1 and OPA1, modulate cellular senescence," The Journal of Biological Chemistry, vol. 282, no. 31, pp. 22977-22983, 2007.

[155] Y. Y. Park, S. Lee, M. Karbowski, A. Neutzner, R. J. Youle, and H. Cho, "Loss of MARCH5 mitochondrial E3 ubiquitin ligase induces cellular senescence through dynamin-related protein 1 and mitofusin 1," Journal of Cell Science, vol. 123, Part 4, pp. 619-626, 2010.

[156] J. Stephan, J. Franke, and A. E. Ehrenhofer-Murray, "Chemical genetic screen in fission yeast reveals roles for vacuolar acidification, mitochondrial fission, and cellular GMP levels in lifespan extension," Aging Cell, vol. 12, no. 4, pp. 574583, 2013.

[157] K. Mao and D. J. Klionsky, "Mitochondrial fission facilitates mitophagy in Saccharomyces cerevisiae," Autophagy, vol. 9, no. 11, pp. 1900-1901, 2013.

[158] K. Mao, K. Wang, X. Liu, and D. J. Klionsky, “The scaffold protein Atg11 recruits fission machinery to drive selective mitochondria degradation by autophagy," Developmental Cell, vol. 26, no. 1, pp. 9-18, 2013.

[159] S. Iqbal and D. A. Hood, "Oxidative stress-induced mitochondrial fragmentation and movement in skeletal muscle myoblasts," American Journal of Physiology. Cell Physiology, vol. 306, no. 12, pp. C1176-C1183, 2014.

[160] S. Wu, F. Zhou, Z. Zhang, and D. Xing, "Mitochondrial oxidative stress causes mitochondrial fragmentation via differential modulation of mitochondrial fission-fusion proteins," The FEBS Journal, vol. 278, no. 6, pp. 941-954, 2011.

[161] B. Kim and Y. S. Song, "Mitochondrial dynamics altered by oxidative stress in cancer," Free Radical Research, vol. 50, no. 10, pp. 1065-1070, 2016.

[162] X. Fan, R. Hussien, and G. A. Brooks, "H2O2-induced mitochondrial fragmentation in $\mathrm{C} 2 \mathrm{C} 12$ myocytes," Free Radical Biology \& Medicine, vol. 49, no. 11, pp. 1646-1654, 2010.

[163] A. Rakovic, A. Grunewald, J. Kottwitz et al., "Mutations in PINK1 and Parkin impair ubiquitination of Mitofusins in human fibroblasts," PloS One, vol. 6, no. 3, article e16746, 2011.

[164] K. F. Cooper, S. Khakhina, S. K. Kim, and R. Strich, "Stressinduced nuclear-to-cytoplasmic translocation of cyclin $\mathrm{C}$ promotes mitochondrial fission in yeast," Developmental Cell, vol. 28, no. 2, pp. 161-173, 2014.

[165] R. Higuchi-Sanabria, W. M. Pernice, J. D. Vevea, D. M. Alessi Wolken, I. R. Boldogh, and L. A. Pon, "Role of asymmetric cell division in lifespan control in Saccharomyces cerevisiae," FEMS Yeast Research, vol. 14, no. 8, pp. 1133-1146, 2014.
[166] J. D. Vevea, T. C. Swayne, I. R. Boldogh, and L. A. Pon, "Inheritance of the fittest mitochondria in yeast," Trends in Cell Biology, vol. 24, no. 1, pp. 53-60, 2014.

[167] J. R. McFaline-Figueroa, J. Vevea, T. C. Swayne et al., "Mitochondrial quality control during inheritance is associated with lifespan and mother-daughter age asymmetry in budding yeast," Aging Cell, vol. 10, no. 5, pp. 885-895, 2011.

[168] W. M. Pernice, J. D. Vevea, and L. A. Pon, "A role for Mfb1p in region-specific anchorage of high-functioning mitochondria and lifespan in Saccharomyces cerevisiae," Nature Communications, vol. 7, article 10595, 2016.

[169] R. Higuchi-Sanabria, J. K. Charalel, M. P. Viana et al., "Mitochondrial anchorage and fusion contribute to mitochondrial inheritance and quality control in the budding yeast Saccharomyces cerevisiae," Molecular Biology of the Cell, vol. 27, no. 5, pp. 776-787, 2016.

[170] R. Erdmann, "Assembly, maintenance and dynamics of peroxisomes," Biochimica et Biophysica Acta (BBA) - Molecular Cell Research, vol. 1863, no. 5, pp. 787-789, 2016.

[171] M. Oku and Y. Sakai, "Peroxisomes as dynamic organelles: autophagic degradation," The FEBS Journal, vol. 277, no. 16, pp. 3289-3294, 2010.

[172] M. Oku and Y. Sakai, "Pexophagy in yeasts," Biochimica et Biophysica Acta (BBA) - Molecular Cell Research, vol. 1863, no. 5, pp. 992-998, 2016.

[173] K. Suzuki, M. Akioka, C. Kondo-Kakuta, H. Yamamoto, and Y. Ohsumi, "Fine mapping of autophagy-related proteins during autophagosome formation in Saccharomyces cerevisiae," Journal of Cell Science, vol. 126, Part 11, pp. 2534-2544, 2013.

[174] K. Suzuki, T. Kirisako, Y. Kamada, N. Mizushima, T. Noda, and Y. Ohsumi, "The pre-autophagosomal structure organized by concerted functions of APG genes is essential for autophagosome formation," The EMBO Journal, vol. 20, no. 21, pp. 5971-5981, 2001.

[175] K. Suzuki, Y. Kubota, T. Sekito, and Y. Ohsumi, "Hierarchy of Atg proteins in pre-autophagosomal structure organization," Genes to Cells, vol. 12, no. 2, pp. 209-218, 2007.

[176] K. Suzuki and Y. Ohsumi, "Current knowledge of the preautophagosomal structure (PAS)," FEBS Letters, vol. 584, no. 7, pp. 1280-1286, 2010.

[177] A. M. Motley, J. M. Nuttall, and E. H. Hettema, “Atg36: the Saccharomyces cerevisiae receptor for pexophagy," Autophagy, vol. 8, no. 11, pp. 1680-1681, 2012.

[178] A. M. Motley, J. M. Nuttall, and E. H. Hettema, "Pex3anchored Atg36 tags peroxisomes for degradation in Saccharomyces cerevisiae," The EMBO Journal, vol. 31, no. 13, pp. 2852-2868, 2012.

[179] C. Tanaka, L. J. Tan, K. Mochida et al., "Hrr25 triggers selective autophagy-related pathways by phosphorylating receptor proteins," The Journal of Cell Biology, vol. 207, no. 1, pp. 91105, 2014.

[180] D. Hoepfner, M. v. d. Berg, P. Philippsen, H. F. Tabak, and E. H. Hettema, "A role for Vpslp, actin, and the Myo2p motor in peroxisome abundance and inheritance in Saccharomyces cerevisiae," The Journal of Cell Biology, vol. 155, no. 6, pp. 979-990, 2001.

[181] A. M. Motley, G. P. Ward, and E. H. Hettema, "Dnm1pdependent peroxisome fission requires Caf4p, Mdv1p and Fis1p," Journal of Cell Science, vol. 121, Part 10, pp. 16331640, 2008. 
[182] K. Kuravi, S. Nagotu, A. M. Krikken et al., "Dynamin-related proteins Vps1p and Dnm1p control peroxisome abundance in Saccharomyces cerevisiae," Journal of Cell Science, vol. 119, Part 19, pp. 3994-4001, 2006.

[183] K. Mao, X. Liu, Y. Feng, and D. J. Klionsky, “The progression of peroxisomal degradation through autophagy requires peroxisomal division," Autophagy, vol. 10, no. 4, pp. 652$661,2014$.

[184] S. Manivannan, R. d. Boer, M. Veenhuis, and I. J. van der Klei, "Lumenal peroxisomal protein aggregates are removed by concerted fission and autophagy events," Autophagy, vol. 9, no. 7, pp. 1044-1056, 2013.

[185] J. M. Nuttall, A. M. Motley, and E. H. Hettema, "Deficiency of the exportomer components Pex1, Pex6, and Pex15 causes enhanced pexophagy in Saccharomyces cerevisiae," Autophagy, vol. 10, no. 5, pp. 835-845, 2014.

[186] S. Manzanares-Estreder, J. Espi-Bardisa, B. Alarcon, A. Pascual-Ahuir, and M. Proft, "Multilayered control of peroxisomal activity upon salt stress in Saccharomyces cerevisiae," Molecular Microbiology, vol. 104, no. 5, pp. 851868, 2017.

[187] M. Honsho, S. Yamashita, and Y. Fujiki, "Peroxisome homeostasis: mechanisms of division and selective degradation of peroxisomes in mammals," Biochimica et Biophysica Acta (BBA) - Molecular Cell Research, vol. 1863, no. 5, pp. 984-991, 2016.

[188] K. Zientara-Rytter and S. Subramani, "Autophagic degradation of peroxisomes in mammals," Biochemical Society Transactions, vol. 44, no. 2, pp. 431-440, 2016.

[189] V. Rogov, V. Dotsch, T. Johansen, and V. Kirkin, "Interactions between autophagy receptors and ubiquitin-like proteins form the molecular basis for selective autophagy," Molecular Cell, vol. 53, no. 2, pp. 167-178, 2014.

[190] M. Nordgren, T. Francisco, C. Lismont et al., "Export-deficient monoubiquitinated PEX5 triggers peroxisome removal in SV40 large T antigen-transformed mouse embryonic fibroblasts," Autophagy, vol. 11, no. 8, pp. 1326-1340, 2015.

[191] J. Zhang, D. N. Tripathi, J. Jing et al., "ATM functions at the peroxisome to induce pexophagy in response to ROS," Nature Cell Biology, vol. 17, no. 10, pp. 1259-1269, 2015.

[192] J. Zhang, J. Kim, A. Alexander et al., "A tuberous sclerosis complex signalling node at the peroxisome regulates mTORC1 and autophagy in response to ROS," Nature Cell Biology, vol. 15, no. 10, pp. 1186-1196, 2013.

[193] D. N. Tripathi, J. Zhang, J. Jing, R. Dere, and C. L. Walker, "A new role for ATM in selective autophagy of peroxisomes (pexophagy)," Autophagy, vol. 12, no. 4, pp. 711-712, 2016.

[194] G. Sargent, T. v. Zutphen, T. Shatseva et al., "PEX2 is the E3 ubiquitin ligase required for pexophagy during starvation," The Journal of Cell Biology, vol. 214, no. 6, pp. 677-690, 2016.

[195] T. Kanki, K. Furukawa, and S. Yamashita, "Mitophagy in yeast: molecular mechanisms and physiological role," Biochimica et Biophysica Acta (BBA) - Molecular Cell Research, vol. 1853, no. 10, Part B, pp. 2756-2765, 2015.

[196] T. Kanki, K. Wang, Y. Cao, M. Baba, and D. J. Klionsky, "Atg32 is a mitochondrial protein that confers selectivity during mitophagy," Developmental Cell, vol. 17, no. 1, pp. 98-109, 2009.

[197] K. Okamoto, N. Kondo-Okamoto, and Y. Ohsumi, "Mitochondria-anchored receptor Atg32 mediates degradation of mitochondria via selective autophagy," Developmental Cell, vol. 17, no. 1, pp. 87-97, 2009.

[198] T. Kanki, Y. Kurihara, X. Jin et al., "Casein kinase 2 is essential for mitophagy," EMBO Reports, vol. 14, no. 9, pp. 788794, 2013.

[199] K. Mao, K. Wang, M. Zhao, T. Xu, and D. J. Klionsky, "Two MAPK-signaling pathways are required for mitophagy in Saccharomyces cerevisiae," The Journal of Cell Biology, vol. 193, no. 4, pp. 755-767, 2011.

[200] Y. Aoki, T. Kanki, Y. Hirota et al., "Phosphorylation of serine 114 on Atg32 mediates mitophagy," Molecular Biology of the Cell, vol. 22, no. 17, pp. 3206-3217, 2011.

[201] H. Abeliovich, M. Zarei, K. T. Rigbolt, R. J. Youle, and J. Dengjel, "Involvement of mitochondrial dynamics in the segregation of mitochondrial matrix proteins during stationary phase mitophagy," Nature Communications, vol. 4, p. 2789, 2013.

[202] S. Bockler and B. Westermann, "Mitochondrial ER contacts are crucial for mitophagy in yeast," Developmental Cell, vol. 28, no. 4, pp. 450-458, 2014.

[203] Y. Kurihara, T. Kanki, Y. Aoki et al., "Mitophagy plays an essential role in reducing mitochondrial production of reactive oxygen species and mutation of mitochondrial DNA by maintaining mitochondrial quantity and quality in yeast," The Journal of Biological Chemistry, vol. 287, no. 5, pp. 3265-3272, 2012.

[204] V. N. Kotiadis, M. R. Duchen, and L. D. Osellame, "Mitochondrial quality control and communications with the nucleus are important in maintaining mitochondrial function and cell health," Biochimica et Biophysica Acta (BBA) General Subjects, vol. 1840, no. 4, pp. 1254-1265, 2014.

[205] G. Twig, A. Elorza, A. J. Molina et al., "Fission and selective fusion govern mitochondrial segregation and elimination by autophagy," The EMBO Journal, vol. 27, no. 2, pp. 433-446, 2008.

[206] G. Twig and O. S. Shirihai, "The interplay between mitochondrial dynamics and mitophagy," Antioxidants \& Redox Signaling, vol. 14, no. 10, pp. 1939-1951, 2011.

[207] T. Kitada, S. Asakawa, N. Hattori et al., "Mutations in the parkin gene cause autosomal recessive juvenile parkinsonism," Nature, vol. 392, no. 6676, pp. 605-608, 1998.

[208] E. M. Valente, P. M. Abou-Sleiman, V. Caputo et al., "Hereditary early-onset Parkinson's disease caused by mutations in PINK1," Science, vol. 304, no. 5674, pp. 11581160, 2004.

[209] H. Shimura, N. Hattori, S. Kubo et al., "Familial Parkinson's disease gene product, parkin, is a ubiquitin-protein ligase," Nature Genetics, vol. 25, no. 3, pp. 302-305, 2000.

[210] J. Park, S. B. Lee, S. Lee et al., "Mitochondrial dysfunction in drosophila PINK1 mutants is complemented by parkin," Nature, vol. 441, no. 7097, pp. 1157-1161, 2006.

[211] I. E. Clark, M. W. Dodson, C. Jiang et al., "Drosophila pink1 is required for mitochondrial function and interacts genetically with parkin," Nature, vol. 441, no. 7097, pp. 11621166, 2006.

[212] E. Deas, H. Plun-Favreau, S. Gandhi et al., "PINK1 cleavage at position A103 by the mitochondrial protease PARL," Human Molecular Genetics, vol. 20, no. 5, pp. 867-879, 2011.

[213] K. Yamano and R. J. Youle, "PINK1 is degraded through the N-end rule pathway," Autophagy, vol. 9, no. 11, pp. 1758-1769, 2013. 
[214] S. M. Jin, M. Lazarou, C. Wang, L. A. Kane, D. P. Narendra, and R. J. Youle, "Mitochondrial membrane potential regulates PINK1 import and proteolytic destabilization by PARL," The Journal of Cell Biology, vol. 191, no. 5, pp. 933-942, 2010.

[215] N. Matsuda, S. Sato, K. Shiba et al., "PINK1 stabilized by mitochondrial depolarization recruits Parkin to damaged mitochondria and activates latent Parkin for mitophagy," The Journal of Cell Biology, vol. 189, no. 2, pp. 211-221, 2010.

[216] D. P. Narendra, S. M. Jin, A. Tanaka et al., "PINK1 is selectively stabilized on impaired mitochondria to activate Parkin," PLoS Biology, vol. 8, no. 1, article e1000298, 2010.

[217] K. Okatsu, M. Kimura, T. Oka, K. Tanaka, and N. Matsuda, "Unconventional PINK1 localization to the outer membrane of depolarized mitochondria drives Parkin recruitment," Journal of Cell Science, vol. 128, no. 5, pp. 964-978, 2015.

[218] K. Okatsu, M. Uno, F. Koyano et al., “A dimeric PINK1containing complex on depolarized mitochondria stimulates Parkin recruitment," The Journal of Biological Chemistry, vol. 288, no. 51, pp. 36372-36384, 2013.

[219] M. Lazarou, S. M. Jin, L. A. Kane, and R. J. Youle, "Role of PINK1 binding to the TOM complex and alternate intracellular membranes in recruitment and activation of the E3 ligase Parkin," Developmental Cell, vol. 22, no. 2, pp. 320-333, 2012.

[220] E. Ziviani, R. N. Tao, and A. J. Whitworth, "Drosophila parkin requires PINK1 for mitochondrial translocation and ubiquitinates mitofusin," Proceedings of the National Academy of Sciences of the United States of America, vol. 107, no. 11, pp. 5018-5023, 2010.

[221] C. Vives-Bauza, C. Zhou, Y. Huang et al., "PINK1-dependent recruitment of Parkin to mitochondria in mitophagy," Proceedings of the National Academy of Sciences of the United States of America, vol. 107, no. 1, pp. 378-383, 2010.

[222] C. Kondapalli, A. Kazlauskaite, N. Zhang et al., "PINK1 is activated by mitochondrial membrane potential depolarization and stimulates Parkin E3 ligase activity by phosphorylating serine 65," Open Biology, vol. 2, no. 5, article 120080, 2012.

[223] K. Shiba-Fukushima, Y. Imai, S. Yoshida et al., "PINK1mediated phosphorylation of the Parkin ubiquitin-like domain primes mitochondrial translocation of Parkin and regulates mitophagy," Scientific Reports, vol. 2, p. 1002, 2012.

[224] L. A. Kane, M. Lazarou, A. I. Fogel et al., "PINK1 phosphorylates ubiquitin to activate Parkin E3 ubiquitin ligase activity," The Journal of Cell Biology, vol. 205, no. 2, pp. 143-153, 2014.

[225] A. Kazlauskaite, C. Kondapalli, R. Gourlay et al., "Parkin is activated by PINK1-dependent phosphorylation of ubiquitin at Ser65," The Biochemical Journal, vol. 460, no. 1, pp. 127139, 2014.

[226] F. Koyano, K. Okatsu, H. Kosako et al., "Ubiquitin is phosphorylated by PINK1 to activate parkin," Nature, vol. 510, no. 7503, pp. 162-166, 2014.

[227] A. Ordureau, S. A. Sarraf, D. M. Duda et al., "Quantitative proteomics reveal a feedforward mechanism for mitochondrial PARKIN translocation and ubiquitin chain synthesis," Molecular Cell, vol. 56, no. 3, pp. 360-375, 2014.

[228] K. Okatsu, F. Koyano, M. Kimura et al., "Phosphorylated ubiquitin chain is the genuine Parkin receptor," The Journal of Cell Biology, vol. 209, no. 1, pp. 111-128, 2015.
[229] K. Shiba-Fukushima, T. Arano, G. Matsumoto et al., "Phosphorylation of mitochondrial polyubiquitin by PINK1 promotes Parkin mitochondrial tethering," PLoS Genetics, vol. 10, no. 12, article e1004861, 2014.

[230] M. E. Gegg, J. M. Cooper, K. Y. Chau, M. Rojo, A. H. Schapira, and J. W. Taanman, "Mitofusin 1 and mitofusin 2 are ubiquitinated in a PINK1/parkin-dependent manner upon induction of mitophagy," Human Molecular Genetics, vol. 19, no. 24, pp. 4861-4870, 2010.

[231] M. E. Gegg and A. H. Schapira, "PINK1-parkin-dependent mitophagy involves ubiquitination of mitofusins 1 and 2: implications for Parkinson's disease pathogenesis," Autophagy, vol. 7, no. 2, pp. 243-245, 2011.

[232] S. A. Sarraf, M. Raman, V. Guarani-Pereira et al., "Landscape of the PARKIN-dependent ubiquitylome in response to mitochondrial depolarization," Nature, vol. 496, no. 7445, pp. 372-376, 2013.

[233] M. Lazarou, D. A. Sliter, L. A. Kane et al., "The ubiquitin kinase PINK1 recruits autophagy receptors to induce mitophagy," Nature, vol. 524, no. 7565, pp. 309-314, 2015.

[234] T. N. Nguyen, B. S. Padman, and M. Lazarou, "Deciphering the molecular signals of PINK1/Parkin mitophagy," Trends in Cell Biology, vol. 26, no. 10, pp. 733-744, 2016.

[235] N. Matsuda, "Phospho-ubiquitin: upending the PINKParkin-ubiquitin cascade," Journal of Biochemistry, vol. 159, no. 4, pp. 379-385, 2016.

[236] M. Frank, S. Duvezin-Caubet, S. Koob et al., "Mitophagy is triggered by mild oxidative stress in a mitochondrial fission dependent manner," Biochimica et Biophysica Acta (BBA) - Molecular Cell Research, vol. 1823, no. 12, pp. 2297-2310, 2012.

[237] T. Murakawa, O. Yamaguchi, A. Hashimoto et al., "Bcl-2-like protein 13 is a mammalian Atg32 homologue that mediates mitophagy and mitochondrial fragmentation," Nature Communications, vol. 6, p. 7527, 2015.

[238] H. Sandoval, P. Thiagarajan, S. K. Dasgupta et al., "Essential role for nix in autophagic maturation of erythroid cells," Nature, vol. 454, no. 7201, pp. 232-235, 2008.

[239] R. L. Schweers, J. Zhang, M. S. Randall et al., "NIX is required for programmed mitochondrial clearance during reticulocyte maturation," Proceedings of the National Academy of Sciences of the United States of America, vol. 104, no. 49, pp. 1950019505, 2007.

[240] L. Liu, D. Feng, G. Chen et al., "Mitochondrial outermembrane protein FUNDC1 mediates hypoxia-induced mitophagy in mammalian cells," Nature Cell Biology, vol. 14, no. 2, pp. 177-185, 2012.

[241] R. J. Wanders, "Peroxisomes in human health and disease: metabolic pathways, metabolite transport, interplay with other organelles and signal transduction," Sub-Cellular Biochemistry, vol. 69, pp. 23-44, 2013.

[242] R. J. Wanders, H. R. Waterham, and S. Ferdinandusse, "Metabolic interplay between peroxisomes and other subcellular organelles including mitochondria and the endoplasmic reticulum," Frontiers in Cell and Development Biology, vol. 3, p. $83,2015$.

[243] R. J. Wanders, J. Komen, and S. Ferdinandusse, "Phytanic acid metabolism in health and disease," Biochimica et Biophysica Acta (BBA) - Molecular and Cell Biology of Lipids, vol. 1811, no. 9, pp. 498-507, 2011. 
[244] J. I. Koepke, C. S. Wood, L. J. Terlecky, P. A. Walton, and S. R. Terlecky, "Progeric effects of catalase inactivation in human cells," Toxicology and Applied Pharmacology, vol. 232, no. 1, pp. 99-108, 2008.

[245] Y. Cohen, Y. A. Klug, L. Dimitrov et al., "Peroxisomes are juxtaposed to strategic sites on mitochondria," Molecular BioSystems, vol. 10, no. 7, pp. 1742-1748, 2014.

[246] M. Mattiazzi Usaj, M. Brloznik, P. Kaferle et al., "Genomewide localization study of yeast Pex11 identifies peroxisome-mitochondria interactions through the ERMES complex," Journal of Molecular Biology, vol. 427, no. 11, pp. 2072-2087, 2015.

[247] B. Kornmann, E. Currie, S. R. Collins et al., “An ERmitochondria tethering complex revealed by a synthetic biology screen," Science, vol. 325, no. 5939, pp. 477-481, 2009.

[248] N. Shai, M. Schuldiner, and E. Zalckvar, "No peroxisome is an island-peroxisome contact sites," Biochimica et Biophysica Acta (BBA) - Molecular Cell Research, vol. 1863, no. 5, pp. 1061-1069, 2016.

[249] J. Fan, X. Li, L. Issop, M. Culty, and V. Papadopoulos, "ACBD2/ECI2-mediated peroxisome-mitochondria interactions in Leydig cell steroid biosynthesis," Molecular Endocrinology, vol. 30, no. 7, pp. 763-782, 2016. 


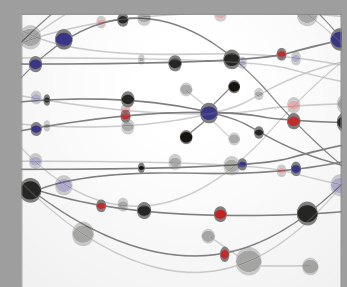

The Scientific World Journal
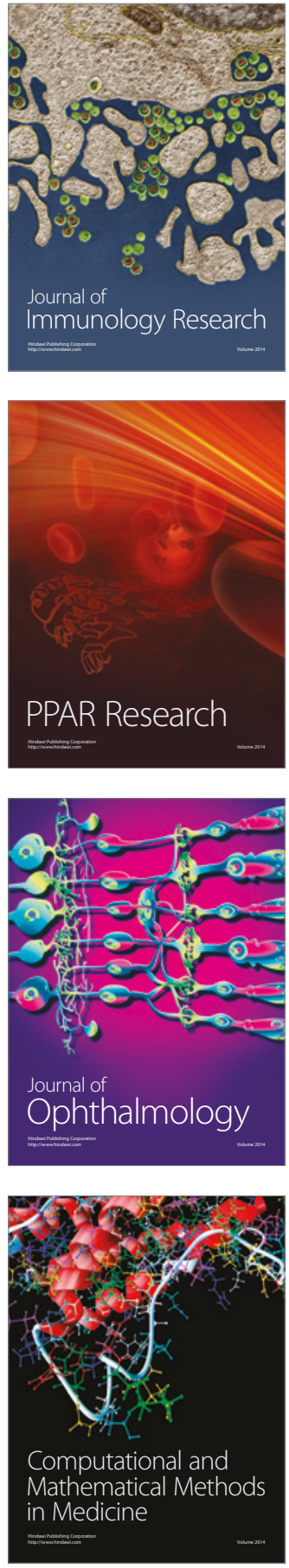

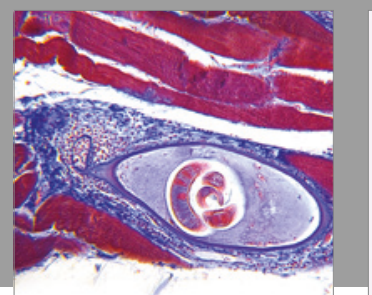

Gastroenterology Research and Practice
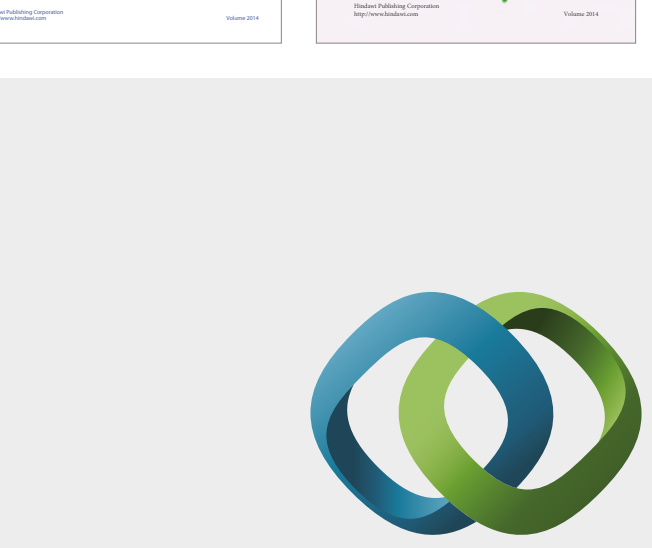

\section{Hindawi}

Submit your manuscripts at

https://www.hindawi.com
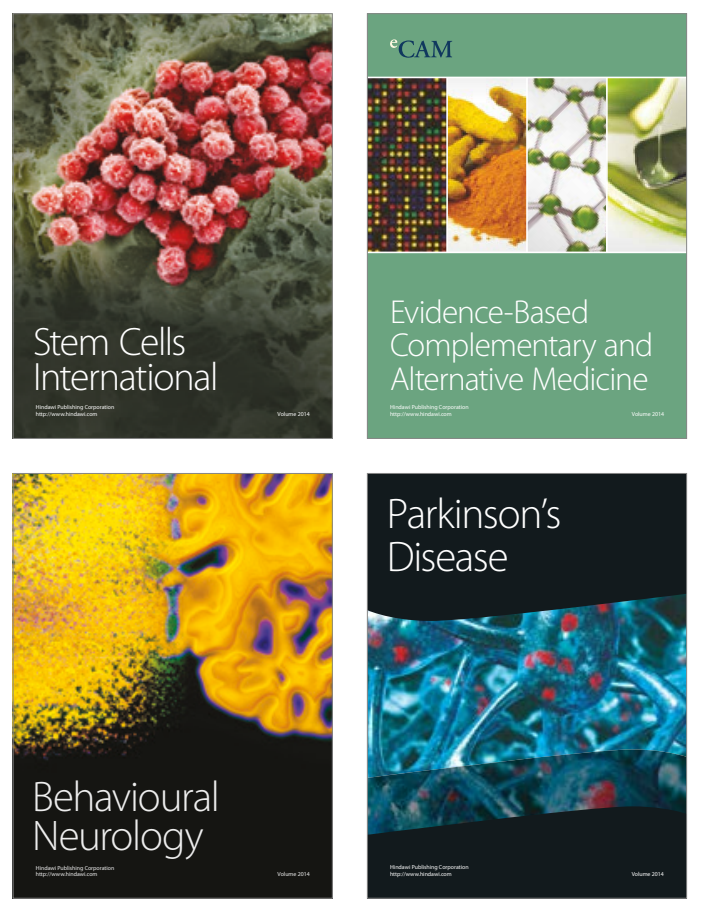
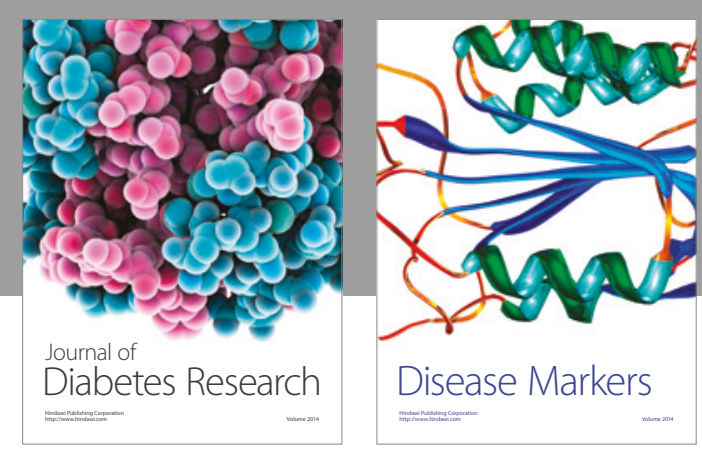

Disease Markers
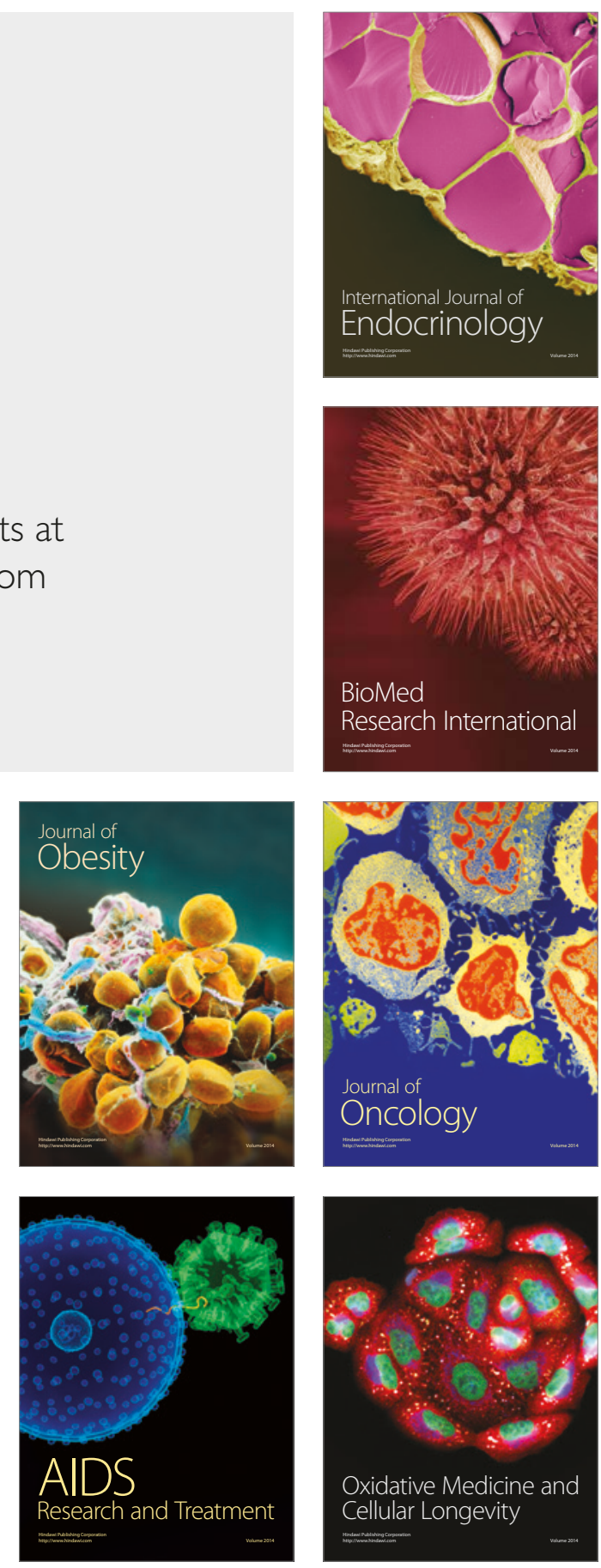